\title{
Antisolar differential rotation with surface lithium enrichment on the single K-giant V1192 Orionis ${ }^{\star}$
}

\author{
Zs. Kővári ${ }^{1}$, K. G. Strassmeier ${ }^{2}$, T. A. Carroll ${ }^{2}$, K. Oláh ${ }^{1}$, L. Kriskovics ${ }^{1}$, E. Kővári ${ }^{3}$, O. Kovács ${ }^{1,3}$, K. Vida ${ }^{1}$, \\ T. Granzer ${ }^{2}$, and M. Weber ${ }^{2}$ \\ 1 Konkoly Observatory, Research Centre for Astronomy and Earth Sciences, Hungarian Academy of Sciences, \\ Konkoly Thege út 15-17, 1121 Budapest, Hungary \\ e-mail: kovari@konkoly.hu \\ 2 Leibniz-Institute for Astrophysics Potsdam (AIP), An der Sternwarte 16, 14482 Potsdam, Germany \\ 3 Eötvös University, Department of Astronomy, Pf. 32, 1518 Budapest, Hungary
}

Received 4 May 2017 / Accepted 1 August 2017

\begin{abstract}
Context. Stars with about 1-2 solar masses at the red giant branch (RGB) represent an intriguing period of stellar evolution, i.e. when the convective envelope interacts with the fast-rotating core. During these mixing episodes freshly synthesized lithium can come up to the stellar surface along with high angular momentum material. This high angular momentum may alter the surface rotation pattern. Aims. The single rapidly rotating K-giant V1192 Ori is revisited to determine its surface differential rotation, lithium abundance, and basic stellar properties such as a precise rotation period. The aim is to independently verify the antisolar differential rotation of the star and possibly find a connection to the surface lithium abundance.

Methods. We applied time-series Doppler imaging to a new multi-epoch data set. Altogether we reconstructed 11 Doppler images from spectroscopic data collected with the STELLA robotic telescope between 2007-2016. We used our inversion code iMap to reconstruct all stellar surface maps. We extracted the differential rotation from these images by tracing systematic spot migration as a function of stellar latitude from consecutive image cross-correlations.

Results. The position of V1192 Ori in the Hertzsprung-Russell diagram suggests that the star is in the helium core-burning phase just leaving the RGB bump. We measure $A(\mathrm{Li})_{\mathrm{NLTE}}=1.27$, i.e. a value close to the anticipated transition value of 1.5 from $\mathrm{Li}$-normal to Li-rich giants. Doppler images reveal extended dark areas arranged quasi-evenly along an equatorial belt. No cool polar spot is found during the investigated epoch. Spot displacements clearly suggest antisolar surface differential rotation with $\alpha=-0.11 \pm 0.02$ shear coefficient.

Conclusions. The surface Li enrichment and the peculiar surface rotation pattern may indicate a common origin.
\end{abstract}

Key words. stars: activity - stars: imaging - stars: late-type - starspots - stars: individual: V1192 Ori

\section{Introduction}

Stellar magnetic activity manifests itself in cool starspots on the stellar surface and is strongly related to rapid rotation. Although most of the stellar angular momentum is supposed to be transferred to its environment by a wind and consequent magnetic braking on the main sequence, there are examples of evolved red giant branch (RGB) stars that are still rapidly rotating and magnetically active. Most of these are members of close binary systems in which tidal forces maintain fast rotation. Rapidly rotating single giants remain a challenge for angular-momentum evolution theories. There are scenarios that allow a star to keep its angular momentum after the main sequence, such as enhanced mixing and dredge-up episodes (Simon \& Drake 1989; Charbonnel \& Lagarde 2010) or planet engulfment (Privitera et al. 2016b).

After the main sequence, RGB stars of about 1-2 solar masses represent an intriguing episode of stellar evolution. When the deepening convective envelope interacts with the fastrotating core, angular momentum is transported to the surface. The mixing of the envelope material with the hotter layers below infer the decay of light elements. This short evolutionary

* Based on data obtained with the STELLA robotic observatory in Tenerife, an AIP facility jointly operated by AIP and IAC. period called the first dredge up is responsible for the dilution of the surface lithium. Despite the expected low lithium abundance, a handful of low-mass stars in this evolutionary state show lithium enrichment on their surfaces. For intermediate mass $\left(\approx 4-7 M_{\odot}\right)$ stars at the asymptotic giant branch (AGB), the Cameron-Fowler mechanism (Cameron \& Fowler 1971) followed by a transport of the Li to cooler regions can plausibly explain the lithium excess. On the other hand, for lower masses at the RGB this mechanism may not work and other non-standard extra mixing processes are required (cf. Sackmann \& Boothroyd 1992; Charbonnel \& Lagarde 2010). In turn, cool-bottom processes (Wasserburg et al. 1995; Sackmann \& Boothroyd 1999) below the convection zone may also be capable for transporting material to layers hot enough for the Cameron-Fowler mechanism and then returning to the convection zone. An alternative evolutionary model was proposed by de La Reza et al. (1996) in which basically every low-mass K-giant undergoes a short $\left(\approx 10^{5} \mathrm{yr}\right.$ ) Li-rich phase on the RGB (see also de La Reza et al. 1997; Kirby et al. 2016). Extra non-axisymmetric mixing that leads to an inhomogeneous super-granulation pattern on the surface in the form of large cool and warm features was invoked to explain super-meteoritic $\mathrm{Li}$ abundances in Strassmeier et al. (2015). 
So far, differential rotation is found to be solar type for mainsequence stars, but giant stars can exhibit antisolar differential rotation, i.e. the equator rotates more slowly than the poles (Vogt \& Hatzes 1991; Strassmeier et al. 2003; Kővári et al. 2015, etc.). Theoretically, this phenomenon is induced and maintained by strong meridional circulation (Kitchatinov \& Rüdiger 2004). Aurnou et al. (2007) proposed that strong mixing by turbulent convection would be the primary agent for angular momentum equilibration and thus antisolar differential rotation. Detailed numerical simulations by, for example Käpylä et al. (2014) and Gastine et al. (2014), suggest that rapidly rotating stars with a small Rossby number yield solar-like differential rotation, while weakly rotating stars with large Rossby numbers may sustain antisolar differential rotation. On the other hand, only three single rapidly rotating giants are known so far to exhibit antisolar differential rotation, namely DI Psc, DP CVn (Kővári et al. 2013), and V1192 Ori (Strassmeier et al. 2003, the star revisited in this paper).

Interestingly, these stars are listed among the very few fast-rotating RGB stars with unusually high surface lithium abundances (see Table 1 in Charbonnel \& Balachandran 2000), implying a possible connection between the antisolar differential rotation profile and the enhanced surface lithium (Kriskovics et al. 2014). We posit whether it is possible that a yet-unknown mixing mechanism responsible for the lithium enrichment can eventually also alter the surface differential rotation profile to be antisolar. We believe that such fast-rotating, single, RGB stars provide a good opportunity to investigate the relationship between activity, rotation, differential rotation, and surface lithium abundance.

In this paper, we present a time-series Doppler imaging study of the single rapidly rotating ( $P_{\text {rot }} \approx 28$ days) K-giant V1192 Ori (=HD 31993), based on spectroscopic observations from the STELLA robotic observatory in Tenerife (Strassmeier et al. 2010). The star was found to have strong CaII H\&K emission and was classified as a K2 giant (Bidelman \& MacConnell 1973; Fekel et al. 1986; Strassmeier et al. 1990) with an unusually high $v \sin i$ value of $31 \mathrm{~km} \mathrm{~s}^{-1}$ (see also Fekel 1997; Fekel \& Balachandran 1993). V1192 Ori was catalogued as Lirich according to its lithium abundance, which is substantially larger than expected for an ordinary K giant (Fekel 1988). The non-local thermodynamic equlibrium (NLTE) lithium abundance for V1192 Ori was measured as $A(\mathrm{Li})=1.4 \pm 0.2$ (Fekel \& Balachandran 1993) on the usual logarithmic scale, i.e. just at the border of $A(\mathrm{Li}) \geq 1.5$ between $\mathrm{Li}$-normal and Li-rich giants (cf. Charbonnel \& Balachandran 2000). Castilho et al. (2000) found $A(\mathrm{Li})$ of 1.7 assuming local thermodynamic equlibrium (LTE), and 1.8 with NLTE correction. More recently, Rebull et al. (2015) quoted the star as Li-normal with $A(\mathrm{Li})=$ 1.26 under NLTE assumption.

V1192 Ori shows a wide range of magnetic-activity indicators from X-ray to infrared (but remains undetected in radio; see Slee et al. 1987). The star was listed as an X-ray source by the Einstein and ROSAT surveys (Gioia et al. 1990; Voges et al. 1999, respectively). International Ultraviolet Explorer (IUE) observations revealed an active UV chromosphere (Fekel \& Balachandran 1993) in accordance with the photospheric light variability (Strassmeier et al. 1997a, 1999), which is attributed to stellar rotation and cool starspots. According to the Zeeman signatures detected by Aurière et al. (2015), V1192 Ori possesses a strong surface magnetic field that is likely produced by an $\alpha \Omega$-type dynamo. The star is also listed in the IRAS (Gezari et al. 1999) and 2MASS (Cutri et al. 2003) infrared point source catalogues.
The first comprehensive photometric and spectroscopic study of V1192 Ori was carried out by (Strassmeier et al. 2003, hereafter Paper I). A rotational period of $\approx 26$ days was derived from the photometric variability which, together with $v \sin i$ of $32 \mathrm{~km} \mathrm{~s}^{-1}$, suggested a minimum radius of $\approx 16 R_{\odot}$ that is consistent with the K2 giant classification. The comparison of the position of V1192 Ori in the Hertzsprung-Russell (H-R) diagram with feasible evolutionary tracks yielded a mass determination of $1.9 M_{\odot}$. In Paper I two Doppler images were presented for consecutive rotation periods showing cool starspots mostly at low to mid-latitudes. Surface differential rotation was investigated by cross-correlating the subsequent maps and yielded antisolar differential rotation. Such observations are relevant constraints for dynamo theory, hence their reliability is of great consequence. Therefore, we revisit V1192 Ori and carry out a new, more detailed Doppler-imaging study from new high-quality spectroscopic data.

The paper is organized as follows. In Sect. 2 we describe the observations and in Sect. 3 provide a more accurate photometric period from the available photometric data. The astrophysical data are summarized in Sect. 4, where we also present a redetermination of the surface lithium abundance. In Sect. 6, we focus on the time-series Doppler imaging. The results are summarized and discussed in Sect. 8.

\section{Observations}

\subsection{Photometry}

Two data sets for V1192 Ori were obtained. The first part of the observations was collected between February 1993-March 1997 (JD 2449 024-2 450 537), while the second part was obtained between March 2007-December 2014 (JD $2454173-$ 2457003). All observations were carried out with the T7 ("Amadeus") $0.75 \mathrm{~m}$ automatic photoelectric telescope (APT) at Fairborn Observatory in southern Arizona (Strassmeier et al. 1997b), which is currently owned and operated by the LeibnizInstitute for Astrophysics Potsdam (AIP); see Granzer et al. (2001) for more details. The two data sets consist of altogether 1312 measurements in Johnson-Cousins $V$ and $I_{C}$. HD 32191 $\left(V=8^{\mathrm{m}} \cdot 520 \pm 0.015, I_{\mathrm{C}}=7 \mathrm{~m} \cdot 287 \pm 0.021\right)$ was used as a comparison star while HD 32073 was used as the check star. Typical data quality is around $6 \mathrm{mmag}$ rms in the first data set but significantly worse in the second because of a defocussing problem. The photometric $V$ data are plotted in Fig. 1.

\subsection{Spectroscopy}

A total of 460 high-resolution echelle spectra were recorded with the $1.2 \mathrm{~m}$ STELLA robotic observatory (Strassmeier et al. 2010) at the Izaña Observatory in Tenerife, Spain, between Jan. 9, 2007 and Feb. 3, 2016. The telescope is equipped with the fibre-fed, fixed-format STELLA Echelle Spectrograph (SES). The spectra cover the full $3900-8800 \AA$ wavelength range with an average spectral resolution of $R=55000$. Further details on the performance of the system and the data reduction procedures can be found in Weber et al. $(2008,2012)$ and Weber \& Strassmeier (2011). Table A.1 gives the log of the SES observations used for the Doppler reconstructions presented in Sect. 6.

\section{Photometric period}

Using the Fourier transformation-based frequency analyser code MuFrAn (Csubry \& Kolláth 2004) we analysed the $V$ data of 
a.

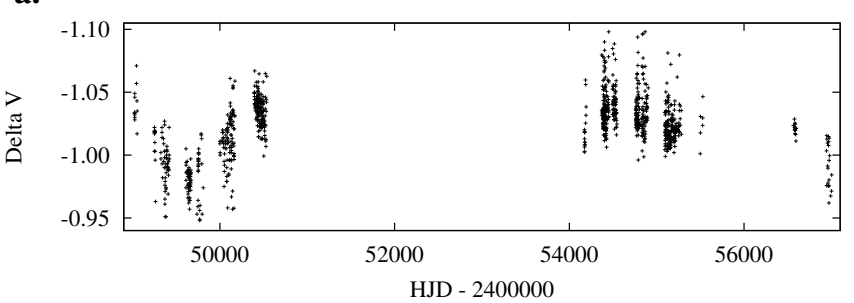

b.

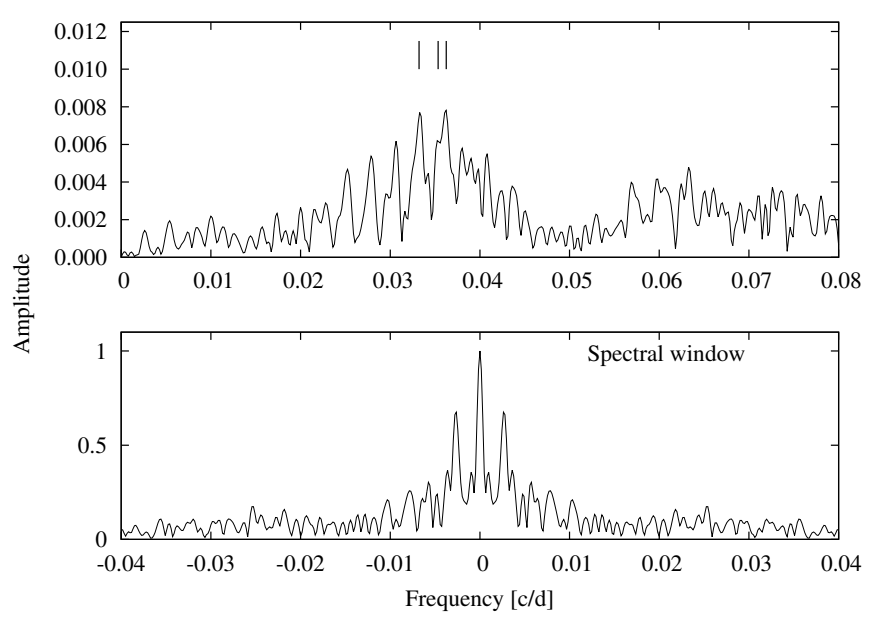

Fig. 1. Panel $a$ : Long-term photometric $V$ data of V1192 Ori observed with the Amadeus APT. Panel b: Amplitude spectrum (top) and spectral window (bottom) from the Johnson $V$ data obtained between 1993 1997. The three most significant period signals are indicated. See text for details.

V1192 Ori to refine the photometric period. In Paper I, only the 1996-1997 APT data were used for the period search, yielding in principle the correct but ambiguous and uncertain period of $25.3 \mathrm{~d}$.

The quality of the new photometric data turned out to be very uneven between the two sets of observations (see Sect. 2.1). We found that the second set from March 2007-December 2014 is rather noisy compared to the first set from February 1993March 1997. Moreover, the rotational variation of V1192 Ori has usually low amplitude. Therefore, we used only the first data part for the period analysis. The resulting amplitude spectrum is shown in Fig. 1. For the refinement of the period we took the three highest peaks and perform a multi-periodic fit. From this the highest amplitude belongs to the $28.3 \mathrm{~d}$ period (middle tick mark in the top panel of Fig. 1). We confirmed this period by consecutively pre-whitening with the three periods. Finally, we settled on $P_{\text {phot }}=28.30 \pm 0.02 \mathrm{~d}$. The other significant periods denoted by the other tick marks are $27.59 \mathrm{~d}$ and $30.06 \mathrm{~d}$. Owing to the crosstalk between the neighbouring peaks, it is not evident that the highest amplitude peak corresponds to the middle tick. Therefore, in Table 1 we listed the frequencies, amplitudes and residuals of 1-, 2-, 3- and 4-component Fourier-fits carried out subsequently. The residuals decrease significantly until the 3-component fit, however, introducing the 4th component yields only marginal improvement. In the 3 - and 4-component fits the highest amplitudes correspond to $\approx 0.0353 \mathrm{c} /$ d, i.e. $\approx 28.3$ days period.

By interpreting the three period peaks as a sign of surface differential rotation, we obtained a surface shear parameter $\Delta P / P$ of $\approx 0.09$. This shear parameter is within the error box of the value derived from consecutive Doppler images in Paper I. From photometric time series alone one cannot determine the sign of
Table 1. Multi-component Fourier-fit parameters.

\begin{tabular}{cccc}
\hline \hline $\begin{array}{c}\text { Frequency } \\
\text { [c/d] }\end{array}$ & Amplitude & Residual & $\begin{array}{c}\text { Residual } \\
\text { difference }\end{array}$ \\
\hline$f_{1}=0.036250$ & 0.00777 & 0.012761 & \\
\hline$f_{1}=0.036214$ & 0.01034 & & \\
$f_{2}=0.032574$ & 0.00819 & 0.011528 & 0.001233 \\
\hline$f_{1}=0.036236$ & 0.00922 & & \\
$f_{2}=0.033289$ & 0.00648 & & \\
$f_{3}=0.035346$ & 0.01090 & 0.010278 & 0.001250 \\
\hline$f_{1}=0.036394$ & 0.00658 & & \\
$f_{2}=0.033197$ & 0.00928 & & \\
$f_{3}=0.035302$ & 0.00940 & & \\
$f_{4}=0.030785$ & 0.00556 & 0.009997 & 0.000281 \\
\hline
\end{tabular}

the surface shear, that is, whether the differential rotation is solar type or antisolar, but see Reinhold \& Arlt (2015) for long-term space photometry.

The new photometric period is longer by $\approx 10 \%$ compared to that from Paper I and is based on a data set that is roughly four times longer. The $2 \mathrm{f}$ harmonic of $13.9 \mathrm{~d}$ in Paper I also indicated a longer period of around $28 \mathrm{~d}$. The second, more noisy part of the data between March 2007-December 2014 yields a $31.8 \mathrm{~d}$ period but with a very low significance. Putting together all the available photometric data results in an $\approx 28 \mathrm{~d}$ period. Therefore, we accept $P_{\text {rot }}=28.30 \mathrm{~d}$ from the first data set as the most feasible and accurate rotation period. Accordingly, for phase calculations we use the following equation:

$\mathrm{HJD}=2450369.2+28.30 \times E$,

where the reference time was chosen arbitrarily (cf. Paper I).

\section{Fundamental parameters}

We redetermined the effective temperature $\left(T_{\text {eff }}\right)$, surface gravity $(\log g)$, metallicity $([\mathrm{Fe} / \mathrm{H}])$, and projected rotational velocity $(v \sin i)$ via the spectrum-synthesis code ParSES (Allende Prieto 2004; Jovanovic et al. 2013) implemented in the standard STELLA-SES data reduction process (Weber et al. 2008). For the synthetic spectra, we determined a microturbulence $\xi_{\text {mic }}$ of $1.25 \mathrm{~km} \mathrm{~s}^{-1}$ by following the empirical relation as was used in the Gaia-ESO survey (Jofré et al. 2014). The radial-tangential macroturbulence $\xi_{\mathrm{mac}}$ of $3 \mathrm{~km} \mathrm{~s}^{-1}$ was taken from Fekel (1997), but see also Paper I. The resulting parameters with their internal standard deviations are listed in Table 2. When compared to the previous values in Paper $\mathrm{I}$, the gravity $\log g$ and $v \sin i$ are only slightly different; these values are still within the small error boxes, but the effective temperature of $4305 \mathrm{~K}$ is lower by $\approx 200 \mathrm{~K}$. However, the new, lower, value is in a better agreement with the colour-index temperature calibration by Worthey \& Lee (2011) when taking $V-I_{\mathrm{C}}=1 \mathrm{~m} 33$ from the long-term photometric data. In Paper I $V-I=1 \mathrm{~m} .21$ was taken from the HIPPARCOS/Tycho catalogue, which would have been in accordance with a temperature of $\approx 4400 \mathrm{~K}$.

The photometric period of $28.3 \mathrm{~d}$ together with the projected rotational velocity of $32.0 \pm 1.5 \mathrm{~km} \mathrm{~s}^{-1}$, and taking $65^{\circ}$ inclination from Paper I, yields a stellar radius $R=19.7_{-2.2}^{+4.4} R_{\odot}$ which, together with $T_{\text {eff }}=4305 \mathrm{~K}$, fits fairly well for a K $2.5 \mathrm{III}$ classification (Dyck et al. 1996; van Belle et al. 1999). In Paper I a parameter study was carried out to select the true inclination by achieving the most homogeneous temperature inversion. For our 
Table 2. Astrophysical properties of V1192 Ori.

\begin{tabular}{|c|c|}
\hline Parameter & Value \\
\hline Spectral type & K2.5 III \\
\hline Distance $_{\text {Gaia }}[\mathrm{pc}]$ & $327_{-28}^{+33}$ \\
\hline$V_{\mathrm{br}}[\mathrm{mag}]$ & $7 \mathrm{~m} \cdot 42^{-28} \pm 0.10$ \\
\hline$(V-I)_{\mathrm{C}, \mathrm{br}}[\mathrm{mag}]$ & $1 \mathrm{~m} .33 \pm 0.03$ \\
\hline$M_{\mathrm{bol}}[\mathrm{mag}]$ & $-1^{\mathrm{m}} .10_{-0.32}^{+0.29}$ \\
\hline Luminosity $\left[L_{\odot}\right]$ & $218^{+73^{-0.52}}$ \\
\hline $\log g[\mathrm{cgs}]$ & $2.39 \pm 0.04$ \\
\hline$T_{\text {eff }}[\mathrm{K}]$ & $4305 \pm 15$ \\
\hline$v \sin i\left[\mathrm{~km} \mathrm{~s}^{-1}\right]$ & $32.0 \pm 1.5$ \\
\hline Rotation period [d] & $28.30 \pm 0.02$ \\
\hline Inclination $\left[{ }^{\circ}\right]$ & $65 \pm 15$ \\
\hline Radius $\left[R_{\odot}\right]$ & $19.7_{-21}^{+4.3}$ \\
\hline Mass $\left[M_{\odot}\right]$ & $1.85 \pm 0.30$ \\
\hline Age $[\mathrm{Gyr}]$ & $1.64 \pm 0.3$ \\
\hline Microturbulence $\left[\mathrm{km} \mathrm{s}^{-1}\right]$ & 1.25 \\
\hline Macroturbulence $\left[\mathrm{km} \mathrm{s}^{-1}\right]$ & 3.0 \\
\hline Metallicity $[\mathrm{Fe} / \mathrm{H}]$ & $-0.08 \pm 0.02$ \\
\hline NLTE Li abundance (log) & $1.27 \pm 0.15$ \\
\hline
\end{tabular}

new spectroscopic data we carried out a similar test with our new inversion code iMap (see Sect. 6.2) and obtained the most acceptable inversions for a range of inclination angles between $50^{\circ}-70^{\circ}$. However, iMap works in a different way and is only of limited use to perform such a parameter search. Thus, we decided to keep the former inclination angle from Paper I assuming a slightly larger error bar of $\pm 15^{\circ}$. From the radius and effective temperature it follows that the luminosity $L=120_{-26}^{+61} L_{\odot}$. When assuming $M_{\mathrm{bol}, \odot}=4.74$ this yields $M_{\mathrm{bol}}=-0.45_{-0.46}^{+0.26}$.

The improved parallax of $3.06 \pm 0.28$ mas from Gaia DR1 (Gaia Collaboration et al. 2016b,a; Lindegren et al. 2016) yields a distance of $327_{-28}^{+33} \mathrm{pc}$, which is $25 \%$ larger compared to the HIPPARCos distance of $238_{-19}^{+83}$ (cf. Paper I). Based on our longterm APT photometry we are able to give a new estimation of $7 \mathrm{~m} .42 \pm 0.10$ for the brightest $V$ magnitude observed so far (see $V_{\mathrm{br}}$ in Table 2). Taking these improved values and assuming an interstellar extinction of $A_{V}=0 \mathrm{~m} \cdot 191$ (Schlegel et al. 1998) together with a bolometric correction of $B C=-0.76$ (Flower 1996) results in $M_{\text {bol }}=-1 \mathrm{~m} \cdot 10_{-0.32}^{+0.29}$. This value and that calculated from $T_{\text {eff }}$ and $R$ agree with each other within their errors. The value $M_{\text {bol }}$ derived from the Gaia parallax converts to a luminosity of $218_{-52}^{+73} L_{\odot}$, which we eventually adopted to find the most plausible position of V1192 Ori in the H-R diagram in Fig. 2. We adopted the PARSEC stellar evolution grid by Bressan et al. $(2012,2013)$, interpolating for $[\mathrm{Fe} / \mathrm{H}]$ of -0.08 $(Z=0.0157)$. From the model grid we obtained a mass of $1.85 \pm 0.3 M_{\odot}$ with an age of $1.64 \pm 0.3 \mathrm{Gyr}$, typical for the epoch just after the RGB bump. This new mass is consistent with the value of $1.9 \pm 0.3 M_{\odot}$ from Paper I. Taking the mass and radius would yield $\log g=2.12_{-0.27}^{+0.17}$, i.e. a bit lower than the adopted value from ParSES. However, such a difference can originate from, for example a somewhat underestimated $V_{\mathrm{br}}$ (cf. Oláh et al. 2014), which would yield lower luminosity, therefore lower mass. Also, a 0.02 dex shift in metallicity yields a mass difference of $\approx 0.1$.

\section{Surface Li abundance}

In Fig. 3 we plot an average Li I-6708 $\AA$ spectrum from summing up seven good-quality $(\mathrm{S} / \mathrm{N}$ of $\approx 200)$ spectra distributed

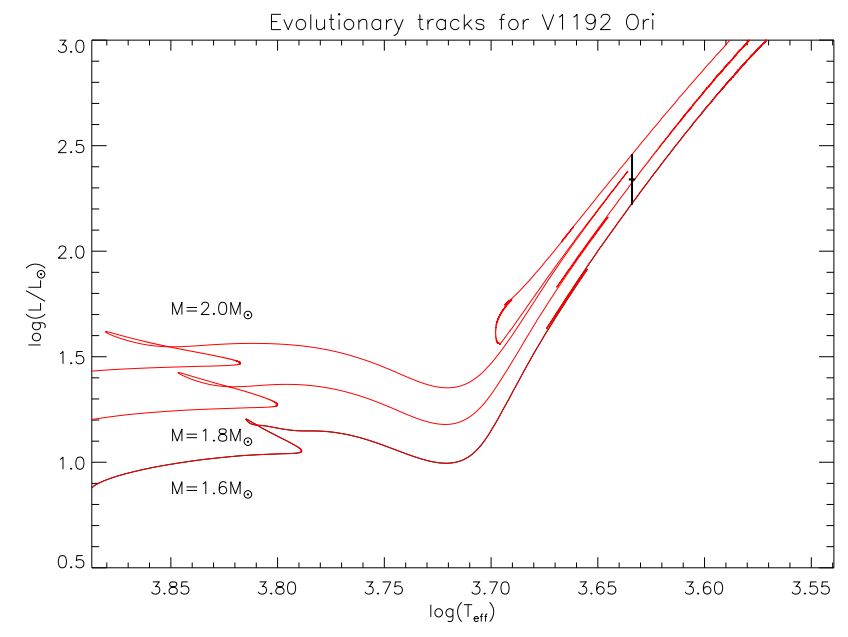

Fig. 2. Position of V1192 Ori (dot) in the H-R diagram. Shown are stellar evolutionary tracks for $1.6,1.8$, and $2.0 M_{\odot}$ from $P A R S E C$, assuming $[\mathrm{Fe} / \mathrm{H}]=-0.08$. The location of V1192 Ori at the RGB indicates a mass of $1.85 M_{\odot}$ and an age of $1.6 \mathrm{Gyr}$.

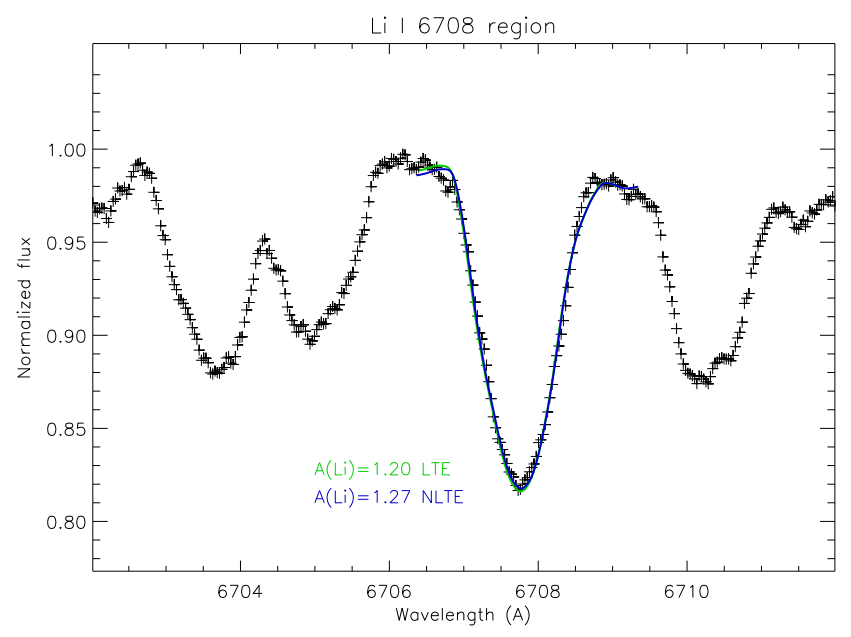

Fig. 3. Observed Li I 6708 A spectral region of V1192 Ori fitted by synthetic Li profiles using LTE (green) and NLTE (blue) approximation.

evenly along all rotation phases in 2008. This spectrum yields $S / N>500: 1$. For the abundance determination, we first employed SME spectral synthesis (Piskunov \& Valenti 2017) with MARCS model atmospheres (Gustafsson et al. 2008) and with LTE approximation. Atomic data were gathered from the Vienna Atomic Line Database (VALD; Kupka et al. 1999). The best fit resulted in $A(\mathrm{Li})=1.20$, i.e. somewhat lower than the value of $1.4 \pm 0.2$ from Fekel \& Balachandran (1993). A $100 \mathrm{~K}$ change in $T_{\text {eff }}$ yields an $\approx 0.15$ dex uncertainty, while a $2 \%$ change in the continuum level yields 0.10 dex.

The NLTE synthesis was also carried out with SME using MARCS model atmospheres but with pre-computed departure coefficients. The fit resulted in $A(\mathrm{Li})_{\mathrm{NLTE}}=1.27$, in good agreement with the recent result by Rebull et al. (2015). We plot the LTE and the NLTE fits together in Fig. 3. Both the LTE and the NLTE approach yields abundances lower than the anticipated limiting value of 1.5 for Li-rich giants. By applying the independent LTE-NLTE abundance correction of Klevas et al. (2016) to our LTE abundance yields $A(\mathrm{Li})_{\mathrm{NLTE}}=1.46$, i.e. also below the nominal 1.5. Thus, we conclude that V1192 Ori is actually not 
Table 3. Temporal distribution of the Doppler images.

\begin{tabular}{ccccccc}
\hline \hline $\begin{array}{c}\text { Observing } \\
\text { run }\end{array}$ & $\begin{array}{c}\text { Doppler } \\
\text { image }\end{array}$ & $\begin{array}{c}\text { Mid-HJD } \\
2450000+\end{array}$ & $\begin{array}{c}\text { Mid-date } \\
\text { yyyy-mm-dd }\end{array}$ & $\begin{array}{c}\text { Number } \\
\text { of spectra }\end{array}$ & $\begin{array}{c}\text { Data coverage } \\
\text { [days] }\end{array}$ & $\begin{array}{c}\text { Data coverage } \\
\text { in } P_{\text {rot }}\end{array}$ \\
\hline 1st & S11 & 4154.266 & $2007-02-22$ & 24 & 26.914 & 0.951 \\
\hline 2nd & S21 & 4353.335 & $2007-09-09$ & 23 & 26.968 & 0.953 \\
& S22 & 4380.154 & $2007-10-06$ & 20 & 26.957 & 0.953 \\
& S23 & 4413.098 & $2007-11-08$ & 27 & 42.950 & 1.518 \\
& S24 & 4470.907 & $2008-01-05$ & 21 & 36.000 & 1.272 \\
& S25 & 4505.701 & $2008-02-09$ & 19 & 45.969 & 1.624 \\
\hline 3rd & S31 & 4785.477 & $2008-11-14$ & 22 & 26.951 & 0.952 \\
& S32 & 4820.173 & $2008-12-19$ & 17 & 30.844 & 1.090 \\
\hline 4th & S41 & 5146.236 & $2009-11-10$ & 19 & 38.924 & 1.375 \\
\hline 5th & S51 & 5616.227 & $2011-02-23$ & 12 & 37.919 & 1.340 \\
\hline 6th & S61 & 7408.699 & $2016-01-21$ & 25 & 26.025 & 0.920 \\
\hline
\end{tabular}
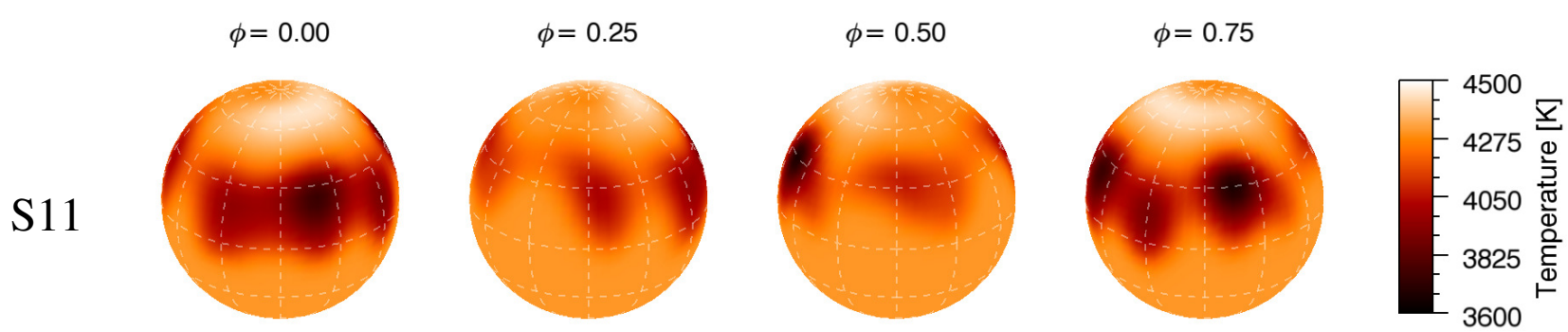

Fig. 4. Doppler image of V1192 Ori for the S11 data set. The corresponding mid-date is 2007-02-22. The spherical surface map is shown in four rotational phases (identified on top) along with the temperature scale.

a bona fide Li-rich giant but a Li-normal star with high surface amounts of Li.

\section{Doppler images for $\mathbf{2 0 0 7 - 2 0 1 6}$}

\subsection{Configuring data subsets for Doppler imaging}

Our spectroscopic data were taken during six observing runs between 2007 and 2016, each providing fairly good sampling for the relatively long rotational phase of 28.3 days. This data set allowed for altogether 11 Doppler reconstructions. Table 3 summarizes the temporal distribution of the Doppler reconstructions over the six runs (see also Table A.1). The second and the third runs were long and continuous enough for obtaining Doppler images for several consecutive stellar rotations, suitable for studying surface differential rotation by tracking short-term spot displacements (see Sect. 7).

\subsection{Image reconstruction code iMap}

Our Doppler reconstruction code iMap performs multi-line inversion simultaneously for a large number of photospheric line profiles (Carroll et al. 2012). For the inversion we selected 40 suitable absorption lines from the 5000-6750 ^ wavelength range by their line depth, blends, continuum level, and their temperature sensitivity (Künstler et al. 2015). Each contributing line is modelled individually and locally and then disk-integrated; finally, all disk-integrated line profiles are averaged to form the mean line profile, which can be compared with each observed mean profile for each observed phase (for more details see Sect. 3 in Carroll et al. 2012).
The iMap code calculates the line profiles by solving the radiative transfer through an artificial neural network (Carroll et al. 2008). Atomic parameters are taken from the VALD database (Kupka et al. 1999). Model atmospheres are taken from Castelli \& Kurucz (2004) and are interpolated for each desired temperature, gravity, and metallicity. Owing to the high workload for computation and modelling, we used LTE radiative transfer instead of spherical model atmospheres. Nevertheless, limitations from neglecting spherical model atmospheres and continuum scattering are compensated by using dense phase coverages (cf. Table 3) and also by using our multi-line approach. For the surface reconstruction iMap uses an iterative regularization based on a Landweber algorithm (Carroll et al. 2012), and therefore no additional constraints are imposed in the image domain. For the inversions we used the same stopping criteria as given by Carroll et al. (2012). According to our tests (see Appendix $\mathrm{A}$ in the aforementioned reference) the iterative regularization (i.e. step size control \& stopping rule) is proved to be enough to converge always to the same image solution. The surface element resolution is set to $5^{\circ} \times 5^{\circ}$.

\subsection{Doppler image reconstructions}

Doppler reconstructions for V1192 Ori reveal a general characteristic; there are cool spots of different sizes and temperature contrasts all around the equatorial regions within a belt extending not higher than $\approx 50^{\circ}$ and a warm azimuthal belt at higher latitudes or partly covering the pole. The individual cool spots change in size considerably from one map to the next. The temperature of the coolest spots is $\approx 700 \mathrm{~K}$ below the effective temperature of the unspotted photosphere. On the other hand, no cool spots at all appear on or near the visible pole during the 

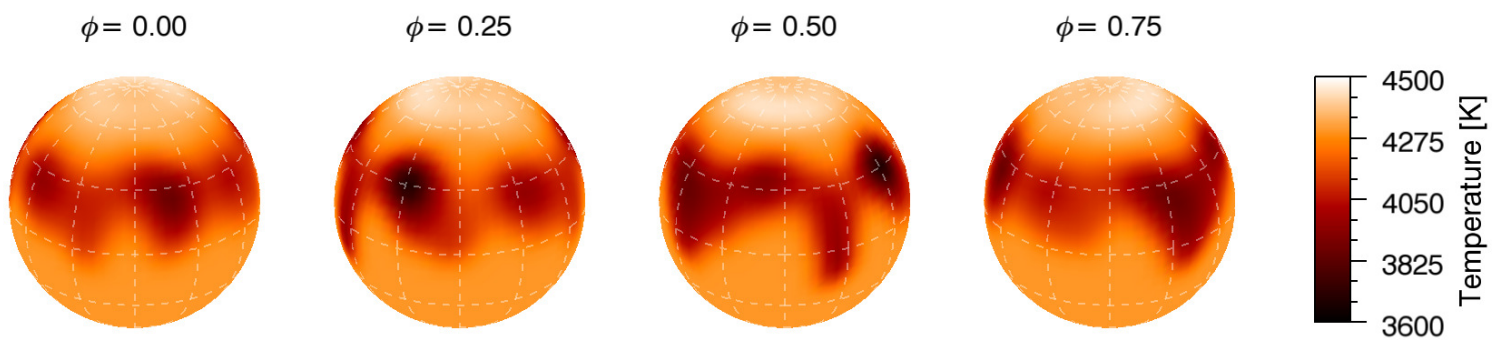

S22
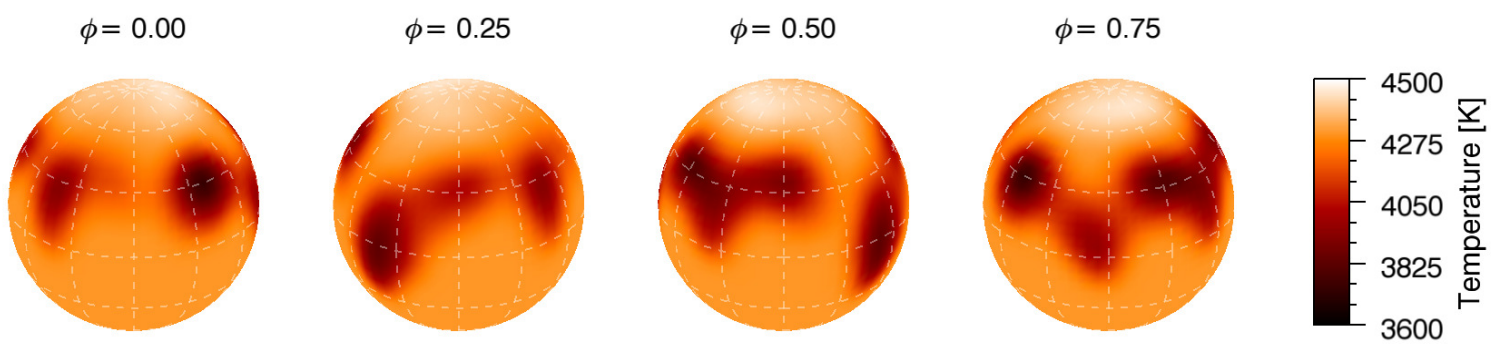

S23
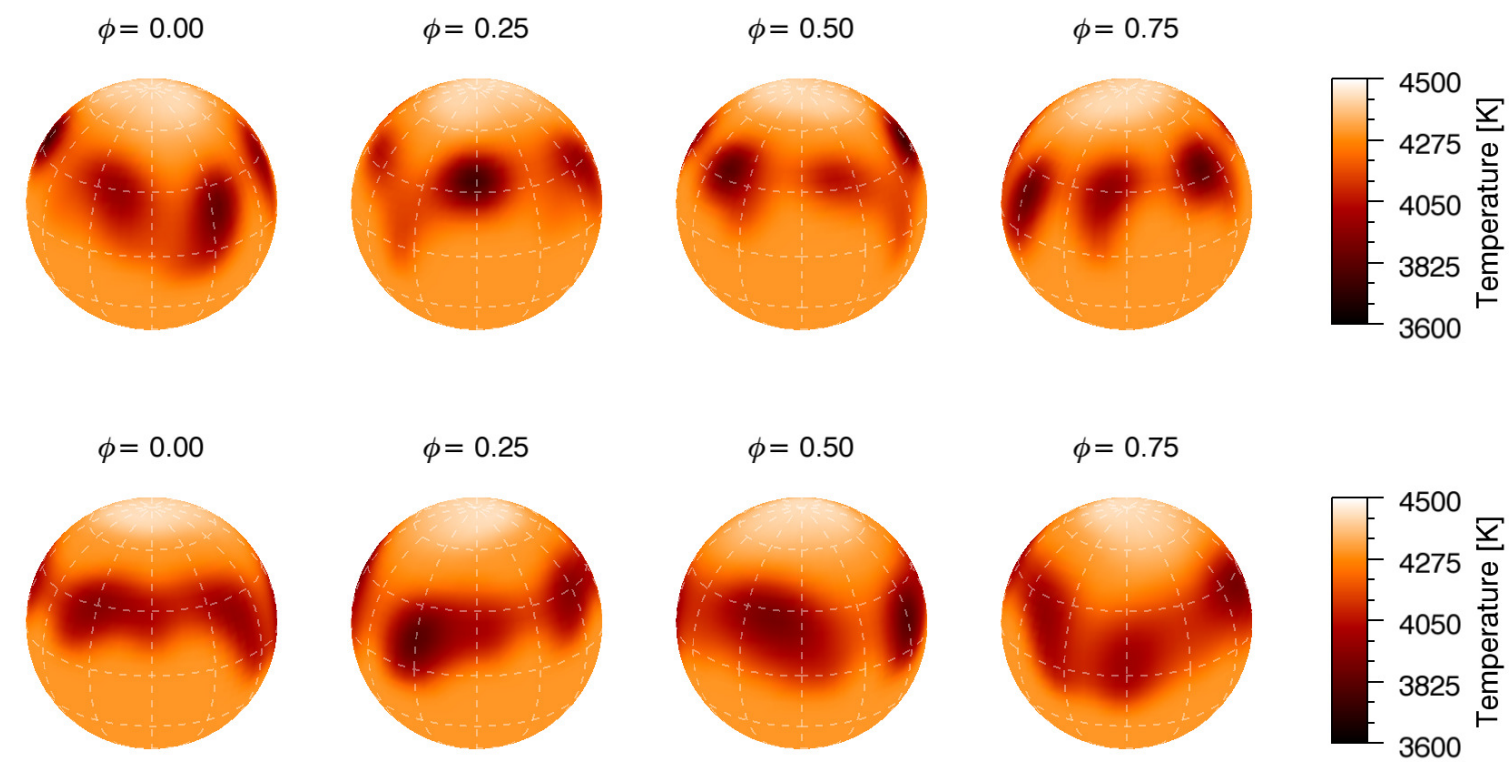

S24
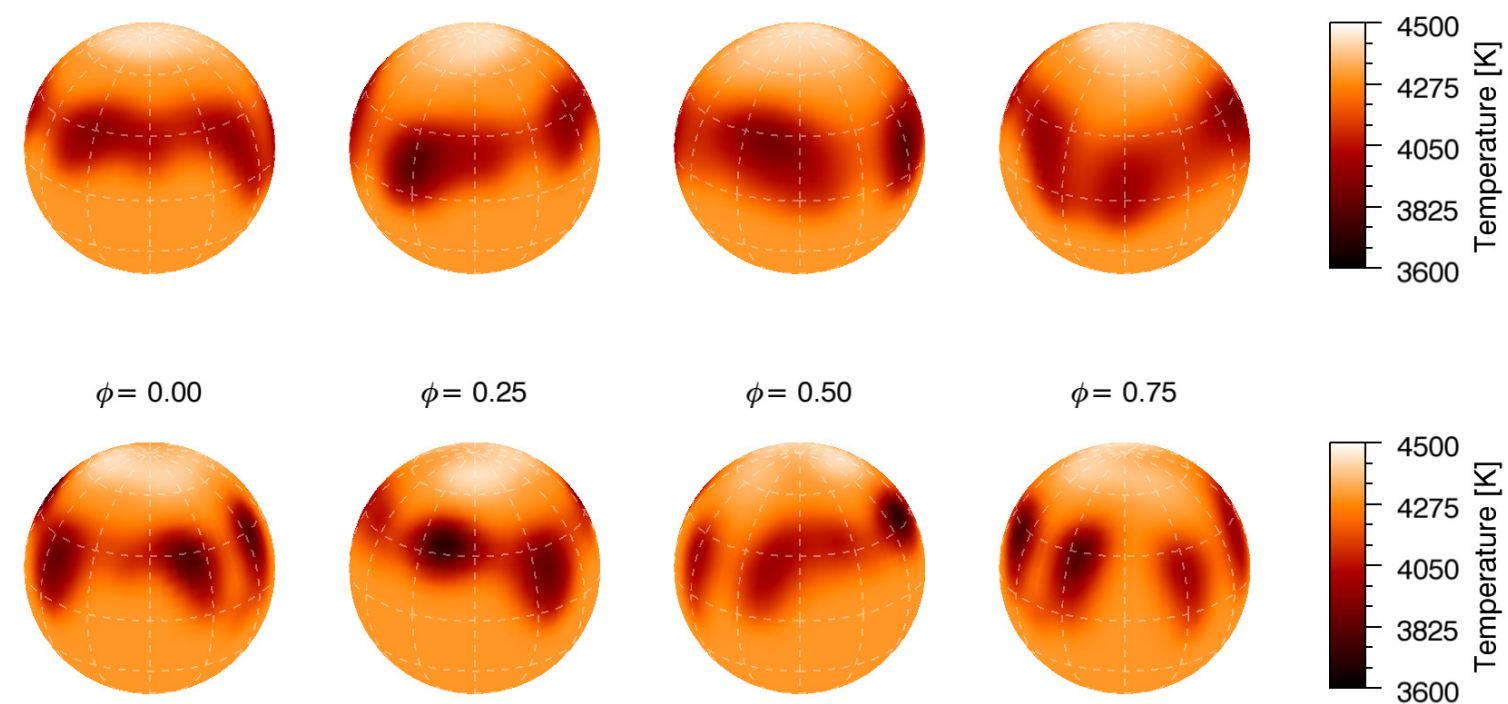

Fig. 5. Doppler images of V1192 Ori for the five data sets S21, S22, S23, S24, and S25. The corresponding mid-dates are 2007-09-09, 2007-10-06, 2007-11-08, 2008-01-05, and 2008-02-09, respectively. Otherwise as in Fig. 4.

time of observations, but the high latitude or even polar patches of $\approx 150 \mathrm{~K}$ warmer regions are seen repeatedly. The overall surface structure with cool spots at lower latitudes and warmer but weakly contrasted features at higher latitudes bears resemblance with the first Doppler images from late 1996 in Paper I, even though those maps revealed lower temperature contrasts. We evaluated the mean error for our temperature maps with a Monte Carlo analysis as described in Carroll et al. (2012) and found a maximum error of $110 \mathrm{~K}$.

First run. The very first season in early 2007 is covered by a single data set that allowed only one surface reconstruction, shown in Fig. 4. It reveals a chain of relatively large, partly 

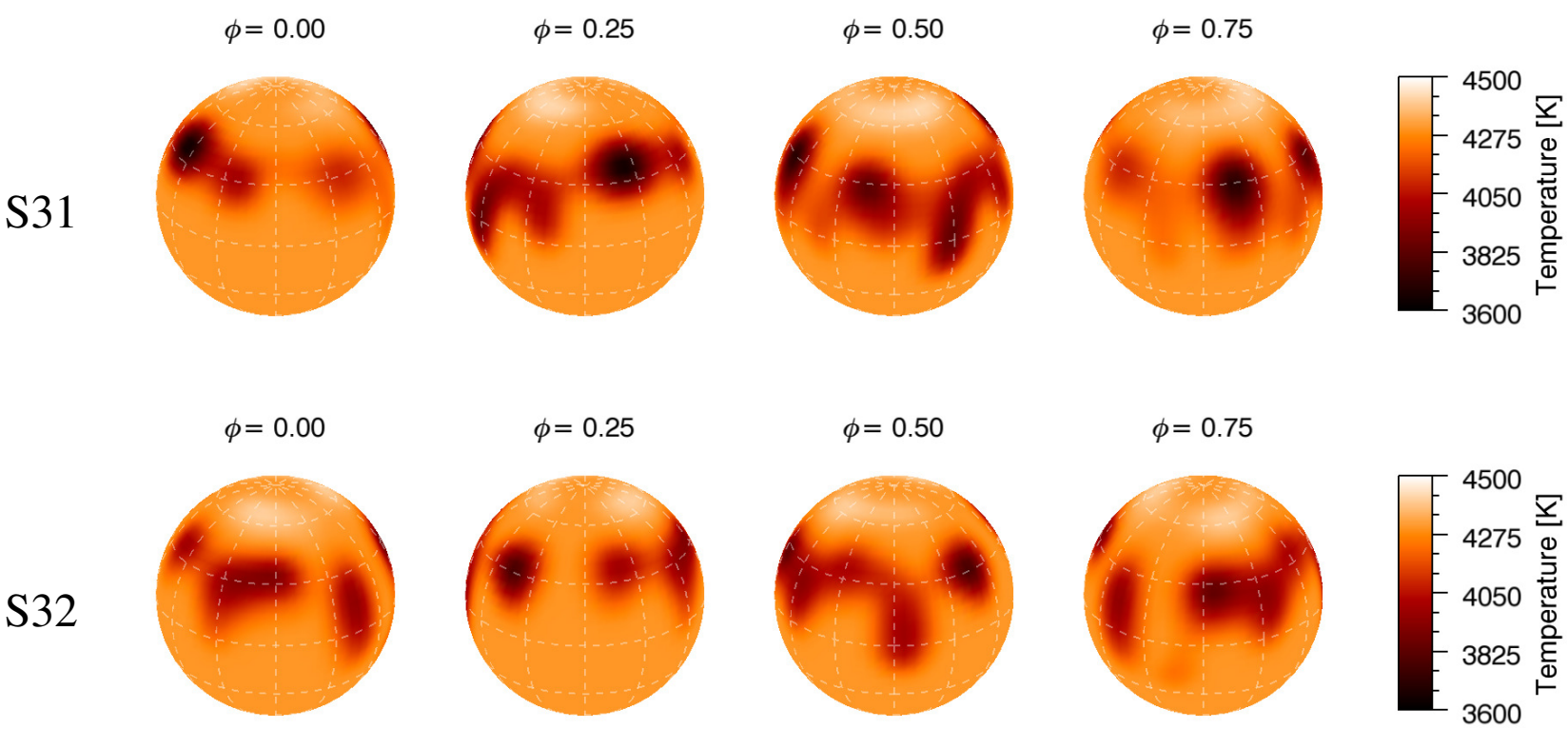

Fig. 6. Doppler images of V1192 Ori for the two data sets S31 and S32. The corresponding mid-dates are 2008-11-14 and 2008-12-19, respectively. Otherwise as in Fig. 4.
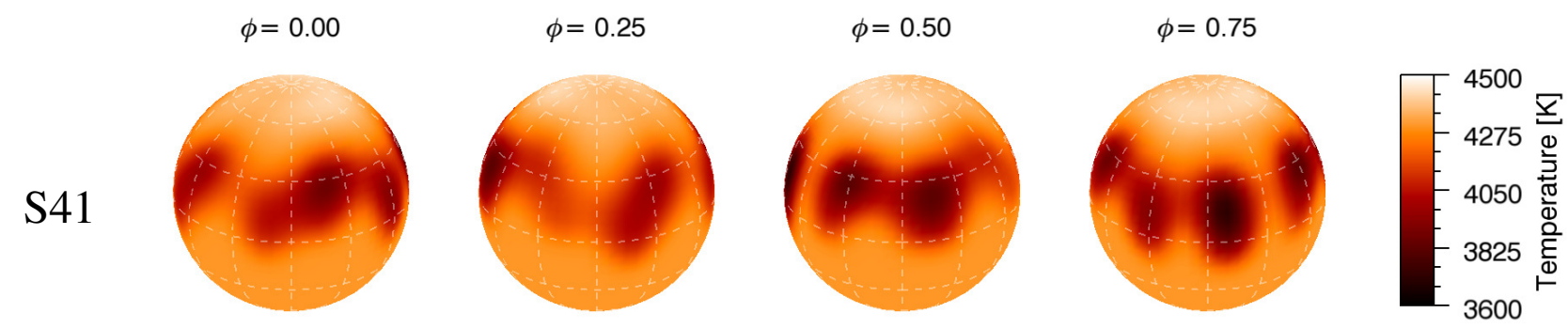

Fig. 7. Doppler image of V1192 Ori for the S41 data set. The corresponding mid-date is 2009-11-10. Otherwise as in Fig. 4.
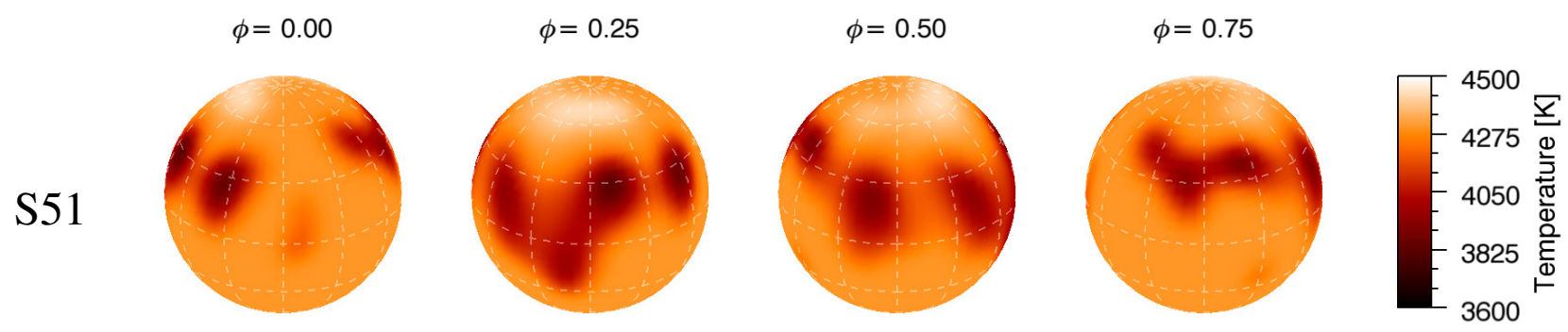

Fig. 8. Doppler image of V1192 Ori for the S51 data set. The corresponding mid-date is 2011-02-23. Otherwise as in Fig. 4.

adjoined spots all around the star. The individual spots have different contrasts but are always cooler than the effective temperature by $\approx 200-600 \mathrm{~K}$. The azimuthal-shaped warm feature extends half around the pole to a longitude that coincides with the most prominent cool spot at lower latitudes, implying that there may be a connection or that the warm feature may only be an artifact or at least raises doubts about its reality.

Second run. The second season from late 2007 to early 2008 is our best-sampled season and provides five maps with a sampling of one map per stellar rotation. Again, as in early 2007, significant changes of the spot arrangement are seen from one map to the next. This is demonstrated in the time series of maps in Fig. 5. Nevertheless, the corresponding dominant spots can easily be tracked on the consecutive maps and, besides some degree of sporadic displacements, the longitudinal tracks already indicate significant differential surface rotation (cf. Sect. 7). This is particularly intriguing because the range of latitudes with spots as surface tracers is comparably narrow. There are also several other noteworthy morphological details, for example a persistent longitudinal gap between spots at around phase 0.4 , or the one latitudinally displaced spot at "southern" latitude. At this point we point out that the Doppler-imaging technique would likely fail to resolve two close-together symmetric latitudinal belts of individual spots, for example as seen on our Sun. Simulations suggested that it likely reconstructs only a single belt placed at the sub-observers latitude (e.g. Rice \& Strassmeier 2000).

Third run. For the third season in late 2008 (Fig. 6), we have another two consecutive maps. These maps again reveal spot 

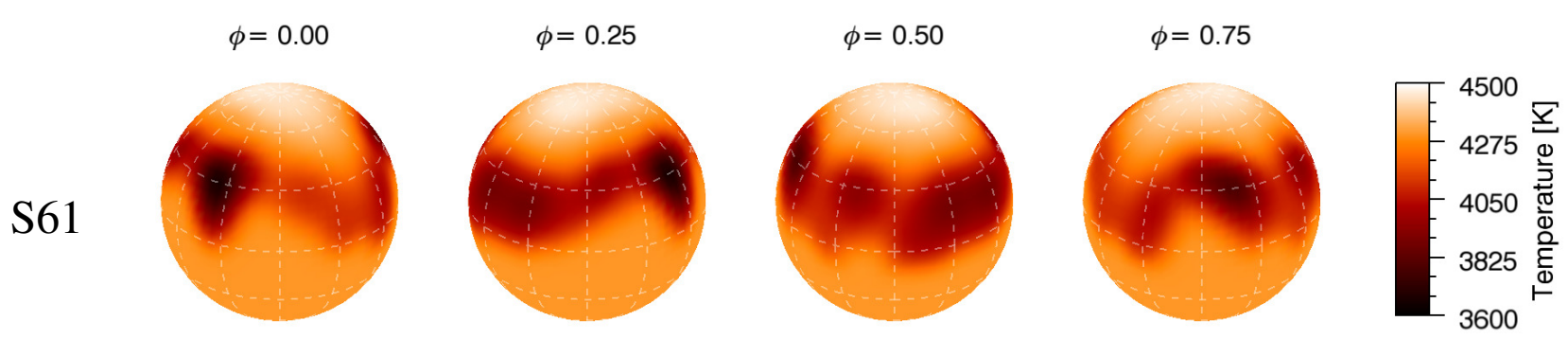

Fig. 9. Doppler image of V1192 Ori for the S61 data set. The corresponding mid-date is 2016-01-21. Otherwise as in Fig. 4.

rearrangements from one rotation to the next, which are partly morphological in nature and partly longitudinal migrations owing to differential surface rotation. The morphological changes were so rapid that there is almost no resemblance between the two maps; this is particularly the case, for example at phase 0.25 in Fig. 6, even though they are from two consecutive rotations. Such rapid variations are also seen on the Sun for particularly active spot groups, while solar plages may not even live as long as one solar rotation. Because the rotation periods of V1192 Ori and the Sun are not so different, $28.3 \mathrm{~d}$ versus $25 \mathrm{~d}$, we may expect that some of the features we are mapping had evolved during the time of observation. If so, only a time average spot would be reconstructed.

Fourth, fifth, and sixth runs. Finally, for the rest of the data, namely from late 2009 (Fig. 7), early 2011 (Fig. 8), and early 2016 (Fig. 9) only one Doppler image per season was possible. Yet, each one shows basically the same morphology, i.e. cool spots at low to mid-latitudes distributed quasi-evenly along all rotational phases and weakly contrasted warm features at very high latitudes, but no cool spot at the pole itself.

\section{Surface differential rotation from time-series Doppler images}

The time-series Doppler images in the second and third observing season allowed us to study the surface differential rotation by means of a cross-correlation analysis of the consecutive maps. Our cross-correlation technique ACCORD (Kővári et al. 2015, and references therein) combines the available information from spot displacements in order to reconstruct the signature of the differential rotation. In the second season from late 2007 to early 2008, we have five consecutive Doppler images (dubbed S21, S22, S23, S24, and S25), while in late 2008, we have two (S31 and S32). Therefore, we are able to create altogether five image pairs (S21-S22, S22-S23, S23-S24, S24-S25, and S31-S32) to be cross-correlated. These correlation maps are combined and the average correlation pattern is fitted with a quadratic rotation law. The result is shown in Fig. 10 and indicates antisolar differential rotation, i.e. on V1192 Ori the rotation rate increases from the equator towards the pole. Its rotation law is expressed in the form $\Omega(\beta)=\Omega_{\mathrm{eq}}\left(1-\alpha \sin ^{2} \beta\right)$, where $\Omega(\beta)$ is the angular velocity at $\beta$ latitude, $\Omega_{\mathrm{eq}}$ is the angular velocity at the equator, while $\alpha$ is the dimensionless surface shear coefficient obtained from $\left(\Omega_{\mathrm{eq}}-\Omega_{\mathrm{pole}}\right) / \Omega_{\mathrm{eq}}$, i.e. the angular velocity difference between the equator and the pole divided by the equatorial velocity. The best fit yields $\Omega_{\mathrm{eq}}=12.695 \pm 0.034^{\circ} / \mathrm{d}$ and $\alpha=-0.11 \pm 0.02$. This can be converted to a lap time of $\approx 260$ days, that is the time the polar regions need to lap the equator by one full rotation.

\section{Summary and discussions}

An extended Doppler imaging study with STELLA during the years 2007 to 2016 yielded 11 new surface image reconstructions, typically one image per stellar rotation. The 11 new Doppler images closely resemble the first Doppler image of V1192 Ori in Paper I from 1996-97 taken with a different telescope-spectrograph combination and with a different inversion code. Its surface spot distribution is well characterized in the sense that cool spots of different sizes and temperatures are always found within a relatively confined latitudinal belt extending up to $\approx 50^{\circ}$ and centred at the sub-observers latitude. No cool spots appear on or near the visible polar region. On the other hand, the individual spots change dynamically, not only from one observing season to the next but from one rotation to the next and possibly even within a single stellar rotation. A warm feature appears consistently at high latitudes as a (partial) azimuthal ring around the pole but we are not certain of its reality. Even though there are now maps spanning 20 years, one cannot identify a clear cyclic behaviour or trend. Long-term variability is certainly present in the photometric light curve on a timescale of 5-10 years (Fig. 1). However, the 10-yr gap in the photometric data from March 1997 to March 2007 prevents us from suggesting any cycle length. We nevertheless refine the rotation period of V1192 Ori from these data to 28.30 days and derive a more reliable set of fundamental stellar parameters by comparing to updated evolutionary tracks. Accordingly, the spectral type of V1192 Ori is found to be K2.5III, i.e. 0.5 subclasses cooler than claimed earlier.

For the time-series Doppler images from the years 20072008, we applied our robust cross-correlation technique and found strong antisolar differential rotation with an $\alpha=-0.11$ surface shear coefficient. This result is in very good agreement with the earlier result of -0.12 from the independent data in Paper I. This is the case even though the $\alpha$ value in Paper I was derived only from a single cross-correlation of two consecutive Doppler maps, and therefore was significantly less robust and had larger error bars. With the present result, we are now confident that V1192 Ori indeed shows strong antisolar differential rotation. Together with DI Psc (Kriskovics et al. 2014) this is the strongest antisolar shear coefficient measured to date. Such strong shear supports the relation of $|\alpha| \propto P_{\text {rot }}$, i.e. the lower the rotation the stronger the shear (Kôvári \& Oláh 2014). Also, a Rossby number of 0.22 derived for V1192 Ori (Aurière et al. 2015) indicates that an $\alpha \Omega$-type dynamo is operating underneath, i.e. differential rotation is expected to play an important role. Table 4 compares V1192 Ori to other measurements of differential surface rotation. The list is not complete and, for the sake of homogeneity, only results from Doppler-imaging studies applying the cross-correlation technique are listed. 
Table 4. Surface shear parameters obtained by cross-correlation of subsequent Doppler images.

\begin{tabular}{lllll}
\hline \hline Star & Type & $P_{\text {rot }}[\mathrm{d}]$ & $\alpha$ Surface shear & Reference \\
\hline AB Dor & K0V, single & 0.51 & $+0.005 \pm 0.001$ & Donati \& Collier Cameron (1997) \\
LQ Hya & K2V, single & 1.60 & $+0.006 \pm 0.001$ & Kővári et al. (2004) \\
EIEri & G5IV, binary & 1.94 & $+0.036 \pm 0.010$ & Kővári et al. (2009) \\
UZ Lib & K0III, binary & 4.76 & $-0.027 \pm 0.009$ & Vida et al. (2007) \\
HU Vir & K0IV, binary & 10.39 & $-0.029 \pm 0.005$ & Harutyunyan et al. (2016) \\
IL Hya & K0IV, binary & 12.91 & $+0.05 \pm 0.01$ & Kővári et al. (2014) \\
DP CVn & K1III, single & 14.01 & $-0.035 \pm 0.016$ & Kővári et al. (2013) \\
$\zeta$ And & K1III, binary & 17.76 & $+0.055 \pm 0.002$ & Kővári et al. (2012) \\
DI Psc & K1III, single & 18.02 & $-0.083 \pm 0.021$ & Kriskovics et al. (2014) \\
$\sigma$ Gem & K1III, binary & 19.60 & $-0.04 \pm 0.01$ & Kôvári et al. (2015) \\
V2075 Cyg & K1III, binary & 22.62 & $+0.015 \pm 0.003$ & Özdarcan et al. (2016) \\
KU Peg & K2III, single & 23.90 & $+0.040 \pm 0.006$ & Kővári et al. (2016) \\
V1192 Ori & K2.5III, single & 28.30 & $-0.11 \pm 0.02$ & this paper \\
\hline
\end{tabular}

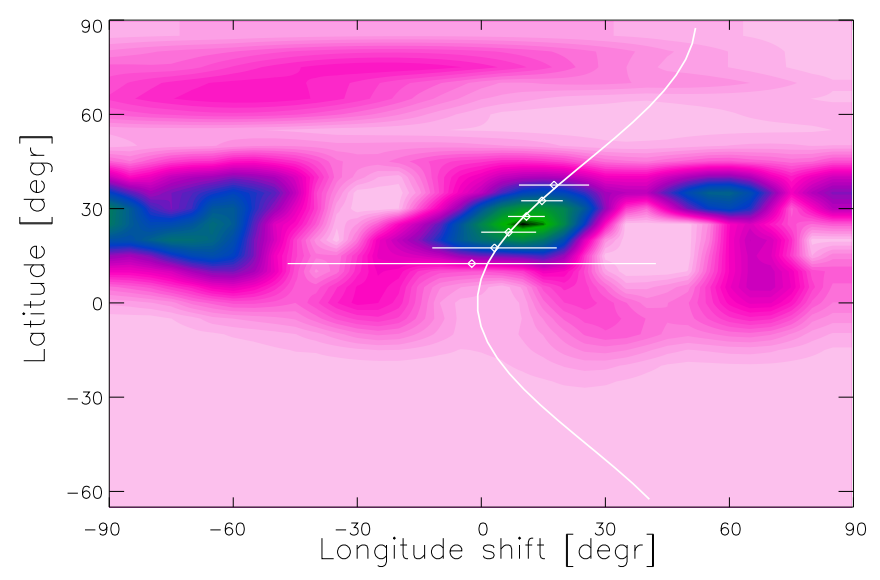

Fig. 10. Average cross-correlation map showing the evidence for surface differential rotation. Darker regions represent better correlation. The average longitudinal cross-correlation functions in $5^{\circ}$ bins are fitted by Gaussian curves. Gaussian peaks are indicated by dots and the corresponding Gaussian widths by horizontal lines. The continuous line is the best fit, suggesting antisolar differential rotation with $P_{\text {eq }}=28.35 \mathrm{~d}$ equatorial period and $\alpha=-0.11$ surface shear.

The unusually fast rotation of a single, evolved star such as V1192 Ori can be explained in various ways, including angular momentum transport from the deep interior (Simon \& Drake 1989). The mass of $1.85 M_{\odot}$ found for V1192 Ori implies a precursor A5 spectral type on the main sequence (Ribas et al. 1997) or even earlier in the case of a significant mass loss. Such a star does not have a deep outer convective zone to maintain a powerful magnetic dynamo that would result in effective magnetic braking on the main sequence (cf. Privitera et al. 2016a). Eventually this means more angular momentum conservation for the post-main sequence evolution, supporting the scenario of mixing up angular momentum. The position in the H-R diagram past the RGB luminosity bump (see Fig. 2) indicates that V1192 Ori has completed Li production at the red-giant bump. According to Charbonnel \& Balachandran (2000) the Li production is followed by an extra mixing phase, interconnecting the $\mathrm{CN}$-burning zone with the convective envelope. Although the Li enrichment is relatively short lived, the extra mixing might explain the surface $\mathrm{Li}$ enrichment together with the peculiar rotation pattern. This is because freshly synthesized $\mathrm{Li}$ comes up to the surface along with high angular momentum material, which can eventually be conveyed towards the poles, resulting in the observed antisolar surface differential rotation (cf. Kitchatinov \& Rüdiger 2004).

This scenario is also compatible with the CameronFowler mechanism (Cameron \& Fowler 1971) of Li production together with the so-called cool-bottom processes (Sackmann \& Boothroyd 1999), which are thought to be responsible for bringing down material from the convective zone and exposing that material to higher temperatures in which partial nuclear fusion ( $\mathrm{H}$ burning) occurs. Then, the Li-rich material is transported back to the convective zone by some circulation or diffusion, wherefrom convective mixing spreads it out towards the surface (see also Busso et al. 2007, and their references). The competing scenario of planet engulfment (cf. Mott et al. 2017, their Sect. 6 and the references therein) explains the existence of the ${ }^{6} \mathrm{Li}$ isotope in stellar atmospheres and may explain the rapid rotation as well, however, it would not easily account for antisolar differential rotation.

Acknowledgements. Authors thank the anonymous referee for his/her valuable comments and suggestions. We thank Dr. Johanna Jurcsik for her helpful notes on deriving the correct rotation period by Fourier transformation. This paper is based on data obtained with the STELLA robotic telescopes in Tenerife, an AIP facility jointly operated by AIP and IAC (https://stella.aip.de/) and by the Amadeus APT jointly operated by AIP and Fairborn Observatory in Arizona. For their continuous support, we are grateful to the ministry for research and culture of the State of Brandenburg (MWFK) and the German federal ministry for education and research (BMBF). Authors from Konkoly Observatory are grateful to the Hungarian National Research, Development and Innovation Office grants OTKA K-109276 and OTKA K-113117, and acknowledge support from the Austrian-Hungarian Action Foundation (OMAA). K.V. is supported by the Bolyai János Research Scholarship of the Hungarian Academy of Sciences. The authors acknowledge the support of the German Deutsche Forschungsgemeinschaft, DFG through projects KO2320/1 and STR645/1. This work has made use of data from the European Space Agency (ESA) mission Gaia (https: //www . cosmos.esa.int/gaia), processed by the Gaia Data Processing and Analysis Consortium (DPAC, https://www. cosmos.esa.int/web/ gaia/dpac/consortium). Funding for the DPAC has been provided by national institutions, in particular the institutions participating in the Gaia Multilateral Agreement.

\section{References}

Allende Prieto, C. 2004, Astron. Nachr., 325, 604

Aurière, M., Konstantinova-Antova, R., Charbonnel, C., et al. 2015, A\&A, 574, A90

Aurnou, J., Heimpel, M., \& Wicht, J. 2007, Icarus, 190, 110

Bidelman, W. P., \& MacConnell, D. J. 1973, AJ, 78, 687

Bressan, A., Marigo, P., Girardi, L., et al. 2012, MNRAS, 427, 127

Bressan, A., Marigo, P., Girardi, L., Nanni, A., \& Rubele, S. 2013, in Eur. Phys. J. Web Conf., 43, 03001 
Busso, M., Wasserburg, G. J., Nollett, K. M., \& Calandra, A. 2007, ApJ, 671, 802

Cameron, A. G. W., \& Fowler, W. A. 1971, ApJ, 164, 111

Carroll, T. A., Kopf, M., \& Strassmeier, K. G. 2008, A\&A, 488, 781

Carroll, T. A., Strassmeier, K. G., Rice, J. B., \& Künstler, A. 2012, A\&A, 548, A95

Castelli, F., \& Kurucz, R. L. 2004, ArXiv e-prints [arXiv: astro-ph/0405087]

Castilho, B. V., Gregorio-Hetem, J., Spite, F., Barbuy, B., \& Spite, M. 2000, A\&A, 364, 674

Charbonnel, C., \& Balachandran, S. C. 2000, A\&A, 359, 563

Charbonnel, C., \& Lagarde, N. 2010, A\&A, 522, A10

Csubry, Z., \& Kolláth, Z. 2004, in SOHO 14 Helio- and Asteroseismology: Towards a Golden Future, ed. D. Danesy, ESA SP, 559, 396

Cutri, R. M., Skrutskie, M. F., van Dyk, S., et al. 2003, VizieR Online Data Catalog: II/246

de La Reza, R., Drake, N. A., \& da Silva, L. 1996, ApJ, 456, L115

de La Reza, R., Drake, N. A., da Silva, L., Torres, C. A. O., \& Martin, E. L. 1997, ApJ, 482, L77

Donati, J.-F., \& Collier Cameron, A. 1997, MNRAS, 291, 1

Dyck, H. M., Benson, J. A., van Belle, G. T., \& Ridgway, S. T. 1996, AJ, 111, 1705

Fekel, F. C. 1988, in ESA SP, 281, 331

Fekel, F. C. 1997, PASP, 109, 514

Fekel, F. C., \& Balachandran, S. 1993, ApJ, 403, 708

Fekel, F. C., Moffett, T. J., \& Henry, G. W. 1986, ApJS, 60, 551

Flower, P. J. 1996, ApJ, 469, 355

Gaia Collaboration (Brown, A. G. A., et al.) 2016a, A\&A, 595, A2

Gaia Collaboration (Prusti, T., et al.) 2016b, A\&A, 595, A1

Gastine, T., Yadav, R. K., Morin, J., Reiners, A., \& Wicht, J. 2014, MNRAS, 438, L76

Gezari, D. Y., Pitts, P. S., \& Schmitz, M. 1999, VizieR Online Data Catalog: II $/ 225$

Gioia, I. M., Maccacaro, T., Schild, R. E., et al. 1990, ApJS, 72, 567

Granzer, T., Reegen, P., \& Strassmeier, K. G. 2001, Astron. Nachr., 322, 325

Gustafsson, B., Edvardsson, B., Eriksson, K., et al. 2008, A\&A, 486, 951

Harutyunyan, G., Strassmeier, K. G., Künstler, A., Carroll, T. A., \& Weber, M. 2016, A\&A, 592, A117

Jofré, P., Heiter, U., Soubiran, C., et al. 2014, A\&A, 564, A133

Jovanovic, M., Weber, M., \& Allende Prieto, C. 2013, Publications de l'Observatoire Astronomique de Beograd, 92, 169

Käpylä, P. J., Käpylä, M. J., \& Brandenburg, A. 2014, A\&A, 570, A43

Kôvári, Zs., \& Oláh, K. 2014, Space Sci. Rev., 186, 457

Kővári, Zs., Strassmeier, K. G., Granzer, T., et al. 2004, A\&A, 417, 1047

Kővári, Zs., Washuettl, A., Foing, B. H., et al. 2009, in 15th Cambridge Workshop on Cool Stars, Stellar Systems, and the Sun, ed. E. Stempels, AIP Conf. Ser., 1094, 676

Kővári, Zs., Korhonen, H., Kriskovics, L., et al. 2012, A\&A, 539, A50

Kővári, Zs., Korhonen, H., Strassmeier, K. G., et al. 2013, A\&A, 551, A2

Kővári, Zs., Kriskovics, L., Oláh, K., et al. 2014, in Magnetic Fields throughout Stellar Evolution, eds. P. Petit, M. Jardine, \& H. C. Spruit, IAU Symp., 302, 379
Kővári, Zs., Kriskovics, L., Künstler, A., et al. 2015, A\&A, 573, A98 Kővári, Zs., Künstler, A., Strassmeier, K. G., et al. 2016, A\&A, 596, A53 Kirby, E. N., Guhathakurta, P., Zhang, A. J., et al. 2016, ApJ, 819, 135 Kitchatinov, L. L., \& Rüdiger, G. 2004, Astron. Nachr., 325, 496

Klevas, J., Kučinskas, A., Steffen, M., Caffau, E., \& Ludwig, H.-G. 2016, A\&A, 586, A156

Kriskovics, L., Kővári, Zs., Vida, K., Granzer, T., \& Oláh, K. 2014, A\&A, 571, A74

Künstler, A., Carroll, T. A., \& Strassmeier, K. G. 2015, A\&A, 578, A101

Kupka, F., Piskunov, N., Ryabchikova, T. A., Stempels, H. C., \& Weiss, W. W. 1999, A\&AS, 138, 119

Lindegren, L., Lammers, U., Bastian, U., et al. 2016, A\&A, 595, A4

Mott, A., Steffen, M., Caffau, E., Spada, F., \& Strassmeier, K. G. 2017, A\&A, 604, A44

Oláh, K., Moór, A., Kővári, Zs., et al. 2014, A\&A, 572, A94

Özdarcan, O., Carroll, T. A., Künstler, A., et al. 2016, A\&A, 593, A123

Piskunov, N., \& Valenti, J. A. 2017, A\&A, 597, A16

Privitera, G., Meynet, G., Eggenberger, P., et al. 2016a, A\&A, 591, A45

Privitera, G., Meynet, G., Eggenberger, P., et al. 2016b, A\&A, 593, A128

Rebull, L. M., Carlberg, J. K., Gibbs, J. C., et al. 2015, AJ, 150, 123

Reinhold, T., \& Arlt, R. 2015, A\&A, 576, A15

Ribas, I., Jordi, C., Torra, J., \& Gimenez, A. 1997, A\&A, 327, 207

Rice, J. B., \& Strassmeier, K. G. 2000, A\&AS, 147, 151

Sackmann, I.-J., \& Boothroyd, A. I. 1992, ApJ, 392, L71

Sackmann, I.-J., \& Boothroyd, A. I. 1999, ApJ, 510, 217

Schlegel, D. J., Finkbeiner, D. P., \& Davis, M. 1998, ApJ, 500, 525

Simon, T., \& Drake, S. A. 1989, ApJ, 346, 303

Slee, O. B., Nelson, G. J., Stewart, R. T., et al. 1987, MNRAS, 229, 659

Strassmeier, K. G., Fekel, F. C., Bopp, B. W., Dempsey, R. C., \& Henry, G. W. 1990, ApJS, 72, 191

Strassmeier, K. G., Bartus, J., Cutispoto, G., \& Rodono, M. 1997a, A\&AS, 125

Strassmeier, K. G., Boyd, L. J., Epand, D. H., \& Granzer, T. 1997b, PASP, 109, 697

Strassmeier, K. G., Serkowitsch, E., \& Granzer, T. 1999, A\&AS, 140, 29

Strassmeier, K. G., Kratzwald, L., \& Weber, M. 2003, A\&A, 408, 1103

Strassmeier, K. G., Granzer, T., Weber, M., et al. 2010, Adv. Astron., 2010, 19

Strassmeier, K. G., Carroll, T. A., Weber, M., \& Granzer, T. 2015, A\&A, 574, A31

van Belle, G. T., Lane, B. F., Thompson, R. R., et al. 1999, AJ, 117, 521

Vida, K., Kővári, Zs., Švanda, M., et al. 2007, Astron. Nachr., 328, 1078

Voges, W., Aschenbach, B., Boller, T., et al. 1999, A\&A, 349, 389

Vogt, S. S., \& Hatzes, A. P. 1991, in The Sun and Cool Stars. Activity, Magnetism, Dynamos (Berlin: Springer Verlag), eds. I. Tuominen, D. Moss, \& G. Rüdiger, IAU Colloq., 130, Lecture Notes in Physics, 380297

Wasserburg, G. J., Boothroyd, A. I., \& Sackmann, I.-J. 1995, ApJ, 447, L37

Weber, M., \& Strassmeier, K. G. 2011, A\&A, 531, A89

Weber, M., Granzer, T., Strassmeier, K. G., \& Woche, M. 2008, in SPIE Conf. Ser., 7019

Weber, M., Granzer, T., \& Strassmeier, K. G. 2012, in SPIE Conf. Ser., 8451

Worthey, G., \& Lee, H.-C. 2011, ApJS, 193, 1 


\section{Appendix A: Observing log of the spectroscopic data and line profile fits for each individual surface} reconstruction

Table A.1. Observing log of STELLA-SES spectra of V1192 Ori from 2007-2016 and grouping into subsets for Doppler reconstructions.

\begin{tabular}{|c|c|c|c|c|c|c|c|c|c|}
\hline $\mathrm{HJD}^{a}$ & Phase $^{b}$ & Date & $S / N$ & Subset & $\mathrm{HJD}^{a}$ & Phase $^{b}$ & Date & $S / N$ & Subset \\
\hline 4140.464 & 0.260 & 08.02 .2007 & 253 & S11 & 4382.641 & 0.818 & 09.10.2007 & 147 & S22 \\
\hline 4141.454 & 0.295 & 09.02.2007 & 296 & S11 & 4383.644 & 0.853 & 10.10 .2007 & 203 & S22 \\
\hline 4142.454 & 0.331 & 10.02 .2007 & 274 & S11 & 4384.638 & 0.888 & 11.10 .2007 & 132 & S22 \\
\hline 4143.455 & 0.366 & 11.02 .2007 & 298 & S11 & 4387.646 & 0.995 & 14.10 .2007 & 186 & S22 \\
\hline 4144.447 & 0.401 & 12.02 .2007 & 185 & S11 & 4388.658 & 0.030 & 15.10 .2007 & 182 & S22 \\
\hline 4146.456 & 0.472 & 14.02 .2007 & 309 & S11 & 4389.635 & 0.065 & 16.10 .2007 & 183 & S22 \\
\hline 4147.414 & 0.506 & 15.02.2007 & 264 & S11 & 4390.614 & 0.099 & 17.10 .2007 & 74 & S22 \\
\hline 4148.397 & 0.541 & 16.02 .2007 & 267 & & 4392.647 & 0.171 & 19.10 .2007 & 182 & S22 \\
\hline 4150.417 & 0.612 & 18.02 .2007 & 283 & & 4393.646 & 0.207 & 20.10 .2007 & 140 & S22 \\
\hline 4152.458 & 0.684 & 20.02 .2007 & 279 & S11 & 4395.630 & 0.277 & 22.10 .2007 & 123 & $\mathrm{~S} 23$ \\
\hline 4153.458 & 0.719 & 21.02 .2007 & 278 & S11 & 4396.614 & 0.311 & 23.10 .2007 & 137 & S23 \\
\hline 4154.476 & 0.755 & 22.02 .2007 & 252 & S11 & 4398.579 & 0.381 & 25.10 .2007 & 126 & $\mathrm{~S} 23$ \\
\hline 4155.480 & 0.791 & 23.02 .2007 & 275 & S11 & 4399.574 & 0.416 & 26.10 .2007 & 125 & S23 \\
\hline 4156.459 & 0.825 & 24.02 .2007 & 186 & S11 & 4400.698 & 0.456 & 27.10 .2007 & 122 & S23 \\
\hline 4157.460 & 0.861 & 25.02.2007 & 158 & S11 & 4402.601 & 0.523 & 29.10 .2007 & 164 & S23 \\
\hline 4158.460 & 0.896 & 26.02.2007 & 253 & S11 & 4403.584 & 0.558 & 30.10 .2007 & 110 & S23 \\
\hline 4159.459 & 0.931 & 27.02.2007 & 240 & & 4404 & & 31.10 .2 & 162 & 23 \\
\hline 4160.461 & 0.967 & 28.02 .20 & 237 & & 4405. & & 01.11 .2 & 177 & 23 \\
\hline 4162.384 & 0.03 & 02.03 .2 & 323 & & 4406.6 & & 2.11 .2 & 151 & $\mathrm{~S} 23$ \\
\hline 4163.361 & 0.069 & 03.03 .2007 & 262 & S11 & 4412. & 0.878 & 08.11 .2 & 57 & S23 \\
\hline 4164.389 & 0.106 & 04.03 .2007 & 304 & S11 & 4412. & 0.880 & 08.11 .2007 & 79 & S23 \\
\hline 4165.375 & 0.140 & 05.03.2007 & 263 & S11 & 4413.659 & 0.914 & 09.11.2007 & 156 & S23 \\
\hline 4166.378 & 0.176 & 06.03.2007 & 294 & S11 & 4414.550 & 0.945 & & 92 & S23 \\
\hline 4167.378 & 0.211 & 07.03 .2007 & 303 & S11 & 4414.704 & 0.951 & 10.11 .2007 & 121 & $\mathrm{~S} 23$ \\
\hline 4338.707 & 0.265 & 26.08.2007 & 144 & S21 & 4416.545 & 0.016 & 12.11 .2007 & 52 & $\mathrm{~S} 23$ \\
\hline 4339.684 & 0.300 & 27.08.2007 & 136 & S21 & 4416.622 & 0.018 & 12.11 .2007 & 174 & S23 \\
\hline 4340.684 & 0.335 & 28.08 .2007 & 101 & & 4417.562 & 0.052 & 13.11 .2007 & 126 & S23 \\
\hline 4343.685 & 0.441 & 31.08 .20 & 145 & & & & & 121 & 23 \\
\hline 4345.673 & & & 131 & & & & & 117 & S23 \\
\hline 4346.672 & 0.547 & 03.09.2007 & 137 & S21 & 4419.578 & 0.123 & 15.11 .2 & 141 & S23 \\
\hline 4347.674 & 0.582 & 04.09 .2007 & 122 & $\mathrm{~S} 21$ & 4420.574 & 0.158 & 16.11 .2007 & 146 & $\mathrm{~S} 23$ \\
\hline 4349.697 & 0.654 & 06.09.2007 & 151 & S21 & 4421.578 & 0.194 & 17.11.2007 & 144 & S23 \\
\hline 4350.699 & 0.689 & 07.09.2007 & 151 & S21 & 4422.683 & 0.233 & 18.11 .2007 & 148 & S23 \\
\hline 4351.692 & 0.724 & 08.09.2007 & 141 & S21 & 4423.575 & 0.264 & 19.11.2007 & 177 & $\mathrm{~S} 23$ \\
\hline 4352.693 & 0.759 & 09.09.2007 & 162 & S21 & 4437.679 & 0.763 & 03.12 .2007 & 220 & S23 \\
\hline 4353.693 & 0.795 & 10.09 .2007 & 129 & & 4438.580 & 0.794 & 04.12 .2007 & 327 & $\mathrm{~S} 23$ \\
\hline 4354.688 & 0.830 & 11.09 .2007 & 145 & & 4450.4 & & & 395 & S24 \\
\hline 4355.692 & 0.865 & 12.09 .2007 & 155 & & 4459. & 0.530 & & 271 & S24 \\
\hline 4357.683 & 0.936 & 14.09 .2007 & 156 & S21 & 4460.401 & 0.565 & 25.12 .2007 & 330 & S24 \\
\hline 4358.695 & 0.972 & 15.09.2007 & 107 & S21 & 4461.402 & 0.601 & 26.12 .2007 & 352 & S24 \\
\hline 4359.672 & 0.006 & 16.09.2007 & 153 & $\mathrm{~S} 21$ & 4462.394 & 0.636 & 27.12.2007 & 342 & S24 \\
\hline 4360.673 & 0.041 & 17.09.2007 & 137 & S21 & 4463.399 & 0.671 & 28.12.2007 & 327 & S24 \\
\hline 4361.669 & 0.077 & 18.09.2007 & 133 & S21 & 4464.408 & 0.707 & 29.12 .2007 & 356 & S24 \\
\hline 4362.669 & 0.112 & 19.09.2007 & 153 & S21 & 4465.388 & 0.742 & 30.12 .2007 & 351 & S24 \\
\hline 4363.672 & 0.147 & 20.09.2007 & 144 & S21 & 4466.386 & 0.777 & 31.12 .2007 & 344 & S24 \\
\hline 4364.667 & 0.183 & 21.09 .2007 & 131 & & 4467.406 & 0.813 & & 306 & S24 \\
\hline 4365.675 & 0.218 & & 12 & & & & & 328 & S24 \\
\hline 4366.689 & 0.254 & 23.09 .2007 & 137 & S22 & 4469.589 & 0.890 & 04.01 .2008 & 263 & S24 \\
\hline 4367.650 & 0.288 & 24.09 .2007 & 55 & S22 & 4471.443 & 0.956 & 05.01 .2008 & 319 & S24 \\
\hline 4369.656 & 0.359 & 26.09.2007 & 108 & S22 & 4475.452 & 0.097 & 09.01.2008 & 357 & S24 \\
\hline 4370.684 & 0.395 & 27.09.2007 & 149 & S22 & 4479.446 & 0.238 & 13.01 .2008 & 315 & S24 \\
\hline 4371.702 & 0.431 & 28.09.2007 & 166 & S22 & 4481.448 & 0.309 & 15.01.2008 & 334 & S24 \\
\hline 4372.664 & 0.465 & 29.09.2007 & 164 & S22 & 4482.456 & 0.345 & 16.01 .2008 & 325 & S24 \\
\hline 4373.664 & 0.501 & 30.09 .2007 & 139 & S22 & 4483.452 & 0.380 & 17.01.2008 & 344 & S24 \\
\hline 4377.662 & 0.642 & 04.10 .2007 & 138 & S22 & 4484.418 & 0.414 & 18.01.2008 & 203 & S24 \\
\hline 4378.653 & 0.677 & 05.10 .2007 & 126 & S22 & 4485.438 & 0.450 & 19.01 .2008 & 182 & S24 \\
\hline 4379.649 & 0.712 & 06.10 .2007 & 158 & S22 & 4486.470 & 0.487 & 20.01 .2008 & 274 & S24 \\
\hline 4380.641 & 0.747 & 07.10 .2007 & 161 & S22 & 4488.410 & 0.555 & 22.01 .2008 & 89 & S25 \\
\hline
\end{tabular}

Notes. ${ }^{(a)} 2450000+.{ }^{(b)}$ Phases computed using Eq. (1). 
Table A.1. continued.

\begin{tabular}{|c|c|c|c|c|c|c|c|c|c|}
\hline $\mathrm{HJD}^{a}$ & Phase $^{b}$ & Date & $S / N$ & Subset & $\mathrm{HJD}^{a}$ & Phase $^{b}$ & Date & $S / N$ & Subset \\
\hline 4488.481 & 0.558 & 22.01 .2008 & 216 & S25 & 5122.662 & 0.967 & 18.10 .2009 & 299 & S41 \\
\hline 4490.497 & 0.629 & 24.01 .2008 & 308 & $\mathrm{~S} 25$ & 5124.655 & 0.037 & 20.10 .2009 & 149 & S41 \\
\hline 4491.452 & 0.663 & 25.01 .2008 & 270 & $\mathrm{~S} 25$ & 5134.656 & 0.391 & 30.10 .2009 & 292 & S41 \\
\hline 4492.389 & 0.696 & 26.01 .2008 & 67 & S25 & 5135.626 & 0.425 & 31.10 .2009 & 336 & S41 \\
\hline 4494.422 & 0.768 & 28.01 .2008 & 280 & $\mathrm{~S} 25$ & 5139.602 & 0.565 & 04.11 .2009 & 318 & S41 \\
\hline 4495.407 & 0.802 & 29.01 .2008 & 242 & $\mathrm{~S} 25$ & 5140.564 & 0.599 & 05.11 .2009 & 126 & S41 \\
\hline 4496.454 & 0.839 & 30.01 .2008 & 249 & $\mathrm{~S} 25$ & 5142.595 & 0.671 & 07.11 .2009 & 270 & S41 \\
\hline 4499.444 & 0.945 & 02.02 .2008 & 257 & $\mathrm{~S} 25$ & 5143.599 & 0.707 & 08.11 .2009 & 250 & S41 \\
\hline 4501.456 & 0.016 & 04.02 .2008 & 312 & S25 & 5144.595 & 0.742 & 09.11 .2009 & 273 & S41 \\
\hline 4502.457 & 0.052 & 05.02 .2008 & 298 & $\mathrm{~S} 25$ & 5146.583 & 0.812 & 11.11 .2009 & 323 & S41 \\
\hline 4508.460 & 0.264 & 11.02 .2008 & 250 & $\mathrm{~S} 25$ & 5147.555 & 0.846 & 12.11 .2009 & 146 & S41 \\
\hline 4512.461 & 0.405 & 15.02 .2008 & 235 & $\mathrm{~S} 25$ & 5149.594 & 0.919 & 14.11 .2009 & 225 & S41 \\
\hline 4514.462 & 0.476 & 17.02.2008 & 263 & $\mathrm{~S} 25$ & 5154.592 & 0.095 & 19.11.2009 & 251 & S41 \\
\hline 4515.461 & 0.511 & 18.02.2008 & 239 & S25 & 5155.602 & 0.131 & 20.11 .2009 & 279 & S41 \\
\hline 4516.461 & 0.546 & 19.02 .2008 & 220 & $\mathrm{~S} 25$ & 5156.583 & 0.165 & 21.11 .2009 & 159 & S41 \\
\hline 4532.378 & 0.109 & 06.03 .2008 & 326 & $\mathrm{~S} 25$ & 5157.589 & 0.201 & 22.11 .2009 & 268 & $\mathrm{~S} 41$ \\
\hline 4533.379 & 0.144 & 07.03 .2008 & 253 & $\mathrm{~S} 25$ & 5159.653 & 0.274 & 24.11 .2009 & 315 & S41 \\
\hline 4534.379 & 0.179 & 08.03.2008 & 305 & S25 & 5160.591 & 0.307 & 25.11 .2009 & 307 & S41 \\
\hline 4774.642 & 0.669 & 04.11 .2008 & 220 & S31 & 5161.586 & 0.342 & 26.11.2009 & 256 & S41 \\
\hline 4775.595 & 0.703 & 05.11 .2008 & 97 & S31 & 5601.457 & 0.885 & 08.02 .2011 & 72 & S51 \\
\hline 4775.639 & 0.705 & 05.11 .2008 & 128 & S31 & 5603.407 & 0.954 & 10.02 .2011 & 96 & S51 \\
\hline 4775.726 & 0.708 & 05.11 .2008 & 197 & S31 & 5607.468 & 0.098 & 14.02 .2011 & 107 & S51 \\
\hline 4776.644 & 0.740 & 06.11 .2008 & 92 & S31 & 5612.403 & 0.272 & 19.02 .2011 & 114 & S51 \\
\hline 4776.733 & 0.743 & 06.11 .2008 & 117 & S31 & 5615.377 & 0.377 & 22.02 .2011 & 115 & S51 \\
\hline 4777.595 & 0.774 & 07.11 .2008 & 220 & S31 & 5616.374 & 0.412 & 23.02.2011 & 113 & S51 \\
\hline 4778.595 & 0.809 & 08.11 .2008 & 209 & S31 & 5617.370 & 0.448 & 24.02 .2011 & 139 & S51 \\
\hline 4779.590 & 0.844 & 09.11 .2008 & 283 & S31 & 5618.370 & 0.483 & 25.02 .2011 & 110 & S51 \\
\hline 4780.586 & 0.879 & 10.11 .2008 & 264 & S31 & 5619.377 & 0.519 & 26.02.2011 & 91 & S51 \\
\hline 4781.670 & 0.918 & 11.11 .2008 & 130 & S31 & 5620.379 & 0.554 & 27.02.2011 & 59 & S51 \\
\hline 4783.586 & 0.985 & 13.11 .2008 & 208 & S31 & 5623.371 & 0.660 & 02.03 .2011 & 122 & S51 \\
\hline 4784.581 & 0.021 & 14.11 .2008 & 260 & S31 & 5639.376 & 0.225 & 18.03 .2011 & 94 & S51 \\
\hline 4785.581 & 0.056 & 15.11 .2008 & 256 & S31 & 7396.439 & 0.312 & 08.01 .2016 & 182 & S61 \\
\hline 4789.583 & 0.197 & 19.11.2008 & 240 & S31 & 7397.478 & 0.349 & 09.01 .2016 & 387 & S61 \\
\hline 4793.594 & 0.339 & 23.11 .2008 & 222 & S31 & 7398.456 & 0.384 & 10.01 .2016 & 364 & S61 \\
\hline 4794.588 & 0.374 & 24.11 .2008 & 229 & S31 & 7399.476 & 0.420 & 11.01 .2016 & 359 & S61 \\
\hline 4795.584 & 0.409 & 25.11 .2008 & 232 & S31 & 7400.476 & 0.455 & 12.01 .2016 & 372 & S61 \\
\hline 4798.671 & 0.518 & 28.11 .2008 & 228 & S31 & 7401.474 & 0.490 & 13.01 .2016 & 196 & S61 \\
\hline 4799.533 & 0.549 & 28.11 .2008 & 225 & S31 & 7402.526 & 0.527 & 14.01 .2016 & 324 & S61 \\
\hline 4800.593 & 0.586 & 30.11 .2008 & 252 & $\mathrm{~S} 31$ & 7403.585 & 0.565 & 16.01 .2016 & 200 & S61 \\
\hline 4801.593 & 0.622 & 01.12 .2008 & 214 & S31 & 7404.503 & 0.597 & 16.01 .2016 & 350 & S61 \\
\hline 4805.597 & 0.763 & 05.12 .2008 & 218 & $\mathrm{~S} 32$ & 7406.523 & 0.669 & 18.01 .2016 & 365 & S61 \\
\hline 4808.589 & 0.869 & 08.12 .2008 & 232 & $\mathrm{~S} 32$ & 7407.477 & 0.702 & 19.01 .2016 & 406 & S61 \\
\hline 4809.663 & 0.907 & 09.12 .2008 & 245 & S32 & 7408.503 & 0.739 & 20.01 .2016 & 395 & S61 \\
\hline 4810.591 & 0.940 & 10.12 .2008 & 173 & $\mathrm{~S} 32$ & 7409.461 & 0.772 & 21.01 .2016 & 361 & S61 \\
\hline 4811.596 & 0.975 & 11.12 .2008 & 222 & S32 & 7410.461 & 0.808 & 22.01 .2016 & 326 & S61 \\
\hline 4812.595 & 0.010 & 12.12 .2008 & 183 & $\mathrm{~S} 32$ & 7411.463 & 0.843 & 23.01 .2016 & 370 & S61 \\
\hline 4814.603 & 0.081 & 14.12 .2008 & 164 & S32 & 7412.485 & 0.879 & 24.01 .2016 & 377 & S61 \\
\hline 4817.606 & 0.187 & 17.12 .2008 & 220 & $\mathrm{~S} 32$ & 7413.499 & 0.915 & 25.01 .2016 & 364 & S61 \\
\hline 4818.494 & 0.219 & 17.12 .2008 & 246 & $\mathrm{~S} 32$ & 7414.464 & 0.949 & 26.01 .2016 & 405 & S61 \\
\hline 4819.470 & 0.253 & 18.12 .2008 & 141 & S32 & 7415.463 & 0.985 & 27.01 .2016 & 390 & S61 \\
\hline 4822.479 & 0.360 & 21.12.2008 & 234 & S32 & 7416.489 & 0.021 & 28.01 .2016 & 187 & S61 \\
\hline 4827.430 & 0.535 & 26.12 .2008 & 65 & $\mathrm{~S} 32$ & 7418.476 & 0.091 & 30.01 .2016 & 361 & S61 \\
\hline 4828.460 & 0.571 & 27.12 .2008 & 229 & $\mathrm{~S} 32$ & 7419.485 & 0.127 & 31.01 .2016 & 242 & S61 \\
\hline 4829.451 & 0.606 & 28.12 .2008 & 147 & $\mathrm{~S} 32$ & 7420.453 & 0.161 & 01.02 .2016 & 69 & S61 \\
\hline 4834.442 & 0.782 & 02.01.2009 & 293 & S32 & 7421.441 & 0.196 & 02.02 .2016 & 195 & S61 \\
\hline 4835.440 & 0.818 & 03.01 .2009 & 236 & S32 & 7422.464 & 0.232 & 03.02 .2016 & 277 & S61 \\
\hline 4836.441 & 0.853 & 04.01 .2009 & 232 & $\mathrm{~S} 32$ & & & & & \\
\hline
\end{tabular}


Zs. Kővári et al.: Antisolar differential rotation and surface lithium enrichment on V1192 Ori
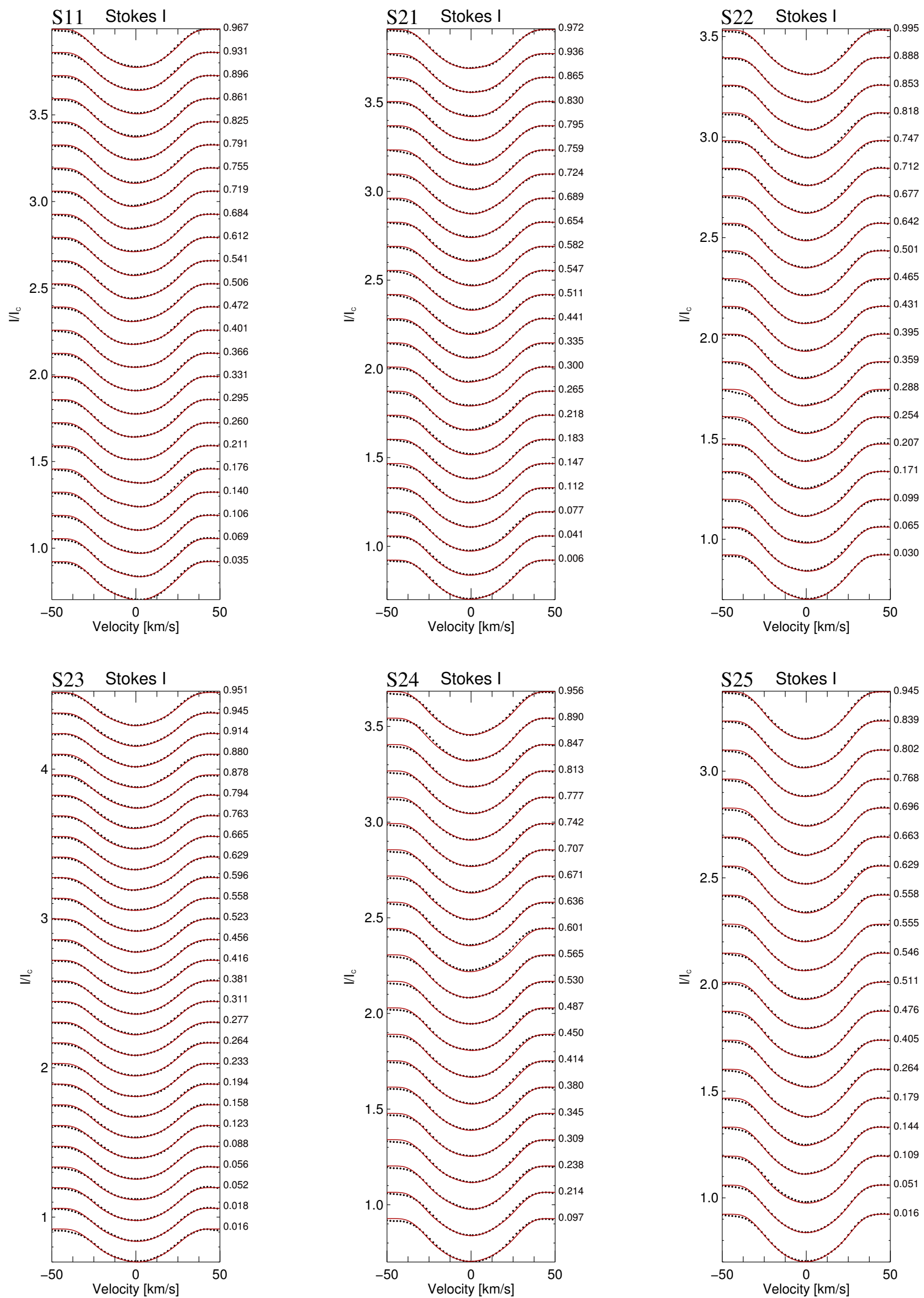

Fig. A.1. Line profile fits for the Doppler reconstructions shown in Figs. 4, 5. The phases of the individual observations are listed on the right side of the panels. 

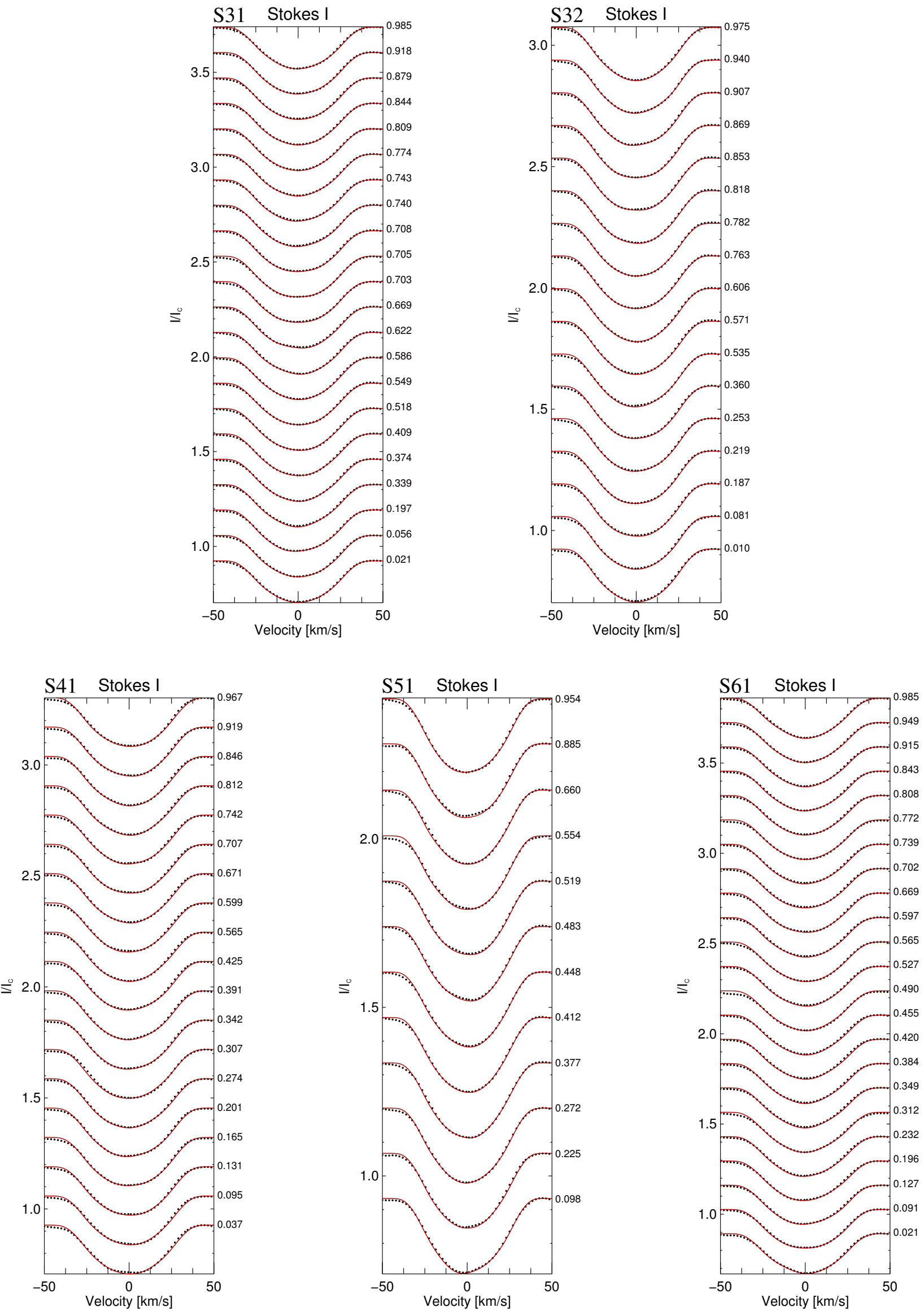

Fig. A.2. Line profile fits for the Doppler reconstructions shown in Figs. 6-9. The phases of the individual observations are listed on the right side of the panels. 